\title{
FOURIER RESTRICTION IN LOW FRACTAL DIMENSIONS
}

\author{
BASSAM SHAYYA
}

\begin{abstract}
Let $S \subset \mathbb{R}^{n}$ be a smooth compact hypersurface with a strictly positive second fundamental form, $E$ be the Fourier extension operator on $S$, and $X$ be a Lebesgue measurable subset of $\mathbb{R}^{n}$. If $X$ contains a ball of each radius, then the problem of determining the range of exponents $(p, q)$ for which the estimate $\|E f\|_{L^{q}(X)} \lesssim\|f\|_{L^{p}(S)}$ holds is equivalent to the restriction conjecture. In this paper, we study the estimate under the following assumption on the set $X$ : there is a number $0<\alpha \leq n$ such that $\left|X \cap B_{R}\right| \lesssim R^{\alpha}$ for all balls $B_{R}$ in $\mathbb{R}^{n}$ of radius $R \geq 1$. On the left-hand side of this estimate, we are integrating the function $|E f(x)|^{q}$ against the measure $\chi_{X} d x$. Our approach consists of replacing the characteristic function $\chi_{X}$ of $X$ by an appropriate weight function $H$, and studying the resulting estimate in three different regimes: small values of $\alpha$, intermediate values of $\alpha$, and large values of $\alpha$. In the first regime, we establish the estimate by using already available methods. In the second regime, we prove a weighted Hölder-type inequality that holds for general nonnegative Lebesgue measurable functions on $\mathbb{R}^{n}$, and combine it with the result from the first regime. In the third regime, we borrow a recent fractal Fourier restriction theorem of $\mathrm{Du}$ and Zhang and combine it with the result from the second regime. In the opposite direction, the results of this paper improve on the Du-Zhang theorem in the range $0<\alpha<n / 2$.
\end{abstract}

\section{INTRODUCTION}

Let $S$ be a smooth compact hypersurface in $\mathbb{R}^{n}$ with a strictly positive second fundamental form, and $\sigma$ be the surface area measure on $S$. The extension operator $E=E_{S}$ on $S$ is defined as

$$
E f(x)=E_{S} f(x)=\widehat{f d \sigma}(x)=\int_{S} e^{-2 \pi i x \cdot \xi} f(\xi) d \sigma(\xi)
$$

for $f \in L^{1}(S)=L^{1}(\sigma)$. The restriction conjecture in harmonic analysis asserts that the operator $E$ is bounded from $L^{p}(S)$ to $L^{q}\left(\mathbb{R}^{n}\right)$ whenever

$$
q>\frac{2 n}{n-1} \quad \text { and } \quad \frac{n-1}{p}+\frac{n+1}{q} \leq n-1 .
$$

This conjecture is proved in the plane, but is largely open in higher dimensions.

There are two important sets of exponents $(p, q)$ satisfying (10): $\{(2, q): q \geq$ $(2 n+2) /(n-1)\}$ and $\{(\infty, q): q>2 n /(n-1)\}$. For the first set of exponents, the restriction conjecture is known to be true in all dimensions $n \geq 2$. In other words, the estimate

$$
\|E f\|_{L^{q}\left(\mathbb{R}^{n}\right)} \lesssim\|f\|_{L^{2}(S)}
$$

holds (uniformly in $f$ ) for $q \geq(2 n+2) /(n-1)$. This result is known in the literature as the Tomas-Stein restriction theorem.

2010 Mathematics Subject Classification. 42B10, 42B20; 28A75. 
For the second set of exponents, there are only partial results. We know that the estimate

$$
\|E f\|_{L^{q}\left(\mathbb{R}^{n}\right)} \lesssim\|f\|_{L^{\infty}(S)}
$$

holds for $q>13 / 4$ when $n=3$ (see [9]), $q>(14 / 5)-(2 / 416515)$ when $n=4$ (see [3] and [23]), $q>2(3 n+1) /(3 n-3)$ when $n \geq 5$ is odd, and $q>2(3 n+2) /(3 n-2)$ when $n \geq 6$ is even (see [10] and [11]). (For a recent improvement in $\mathbb{R}^{3}$, see [20; and in $\mathbb{R}^{n}, n \geq 4$, see [13.)

We also refer the reader to [17] and [8] for the full range of $p$ and $q$ exponents corresponding to Guth's $q>13 / 4$ result in 9 .

Suppose $n \geq 1$ and $0<\alpha \leq n$. For Lebesgue measurable functions $H: \mathbb{R}^{n} \rightarrow$ $[0,1]$, we define

$$
A_{\alpha}(H)=\inf \left\{C: \int_{B\left(x_{0}, R\right)} H(x) d x \leq C R^{\alpha} \text { for all } x_{0} \in \mathbb{R}^{n} \text { and } R \geq 1\right\},
$$

where $B\left(x_{0}, R\right)$ denotes the closed ball in $\mathbb{R}^{n}$ of center $x_{0}$ and radius $R$. We say $H$ is a weight of (fractal) dimension $\alpha$ if $A_{\alpha}(H)<\infty$. We note that $A_{\beta}(H) \leq A_{\alpha}(H)$ if $\beta \geq \alpha$, so we are not really assigning a dimension to the function $H$; the phrase " $H$ is a weight of dimension $\alpha$ " is merely another way for us to say that $A_{\alpha}(H)<\infty$. (The motivation for referring to $\alpha$ as a fractal dimension comes from Sections 4 and 8 below.)

We are interested in weighted restriction estimates of the form

$$
\|E f\|_{L^{q}(H d x)} \lesssim A_{\alpha}(H)^{1 / q}\|f\|_{L^{p}(S)}
$$

that hold uniformly in $f$ and $H$. In other words, the implicit constant in (4) is allowed to depend on the exponents $p$ and $q$, the dimensions $\alpha$ and $n$, and the surface $S$; but must be independent of the functions $f$ on $S$ and the weights $H$ on $\mathbb{R}^{n}$. We shall refer to (4) as a weighted $L^{p}$-based estimate.

One of the main goals of this paper is to prove the following theorem, and then use it in $\mathbb{R}^{n}, n \geq 2$, to obtain new results concerning weighted $L^{2}$-based and $L^{\infty}$ based restriction estimates.

For Lebesgue measurable functions $F: \mathbb{R}^{n} \rightarrow[0, \infty)$, we define

$$
M_{\alpha} F=\left(\sup _{H} \frac{1}{A_{\alpha}(H)} \int F(x)^{\alpha} H(x) d x\right)^{1 / \alpha},
$$

where $H$ ranges over all non-zero weights on $\mathbb{R}^{n}$ of dimension $\alpha$. We prove the following weighted Hölder-type inequality that might be of independent interest.

Theorem 1.1. Suppose $n \geq 1$ and $0<\beta<\alpha \leq n$. Then

$$
M_{\alpha} F \leq M_{\beta} F
$$

for all non-negative Lebesgue measurable functions $F$ on $\mathbb{R}^{n}$.

Before we present the rest of our results, we give a couple of examples that are meant to provide the reader with a quick overview of the main theme of the paper concerning $L^{2}$-based estimates. In both examples, which take place in the plane, $S$ will be the unit circle.

Consider the set $X=\left\{(x, y) \in \mathbb{R}^{2}: x>0\right.$ and $\left.0 \leq y \leq x^{-1 / 4}\right\}$. It is easy to see that the characteristic function $\chi_{X}$ is a weight on $\mathbb{R}^{2}$ of dimension $3 / 4$. We 
want to determine the best range of exponents $q$ for which the following restriction estimate holds:

$$
\|E f\|_{L^{q}(X)} \lesssim\|f\|_{L^{2}(S)} .
$$

To every $R>1$ there is a function $f_{R}$ on $S$ such that $\left\|f_{R}\right\|_{L^{2}(S)} \lesssim R^{-1 / 4}$ and $\left|E f_{R}\right| \gtrsim R^{-1 / 2}$ on the rectangle $[0, R] \times[0, \sqrt{R}]$. The intersection of this rectangle with $X$ contains the rectangle $[0, R] \times\left[0, R^{-1 / 4}\right]$, and hence $\left\|E f_{R}\right\|_{L^{q}(X)}$ $\gtrsim R^{(-1 / 2)+(3 /(4 q))}$. So the exponent $q$ in (6) must satisfy $(-1 / 2)+(3 /(4 q)) \leq-1 / 4$, and so a necessary condition for (6) to hold is $q \geq 3$, which is far from the sufficient condition $q \geq 6$ guaranteed by (2). Even the $L^{\infty}$-based estimate (3) only gives the sufficient condition $q>4$ in the plane.

In the second example, we consider the set $Y=\cup_{l=1}^{\infty} \mathbb{R} \times\left[l^{2}, 1+l^{2}\right]$, and we observe that the characteristic function $\chi_{Y}$ is a weight on $\mathbb{R}^{2}$ of dimension $3 / 2$. Again, we want to determine the best range of exponents $q$ for which the following restriction estimate holds:

$$
\|E f\|_{L^{q}(Y)} \lesssim\|f\|_{L^{2}(S)} .
$$

For $R>1$, let $f_{R}$ be the same function on $S$ that was defined during the first example. Then $\left|E f_{R}\right| \gtrsim R^{-1 / 2}$ on every rectangle $[0, R] \times\left[l^{2}, 1+l^{2}\right]$ with $l^{2} \leq \sqrt{R}$. Since there are $\sim R^{1 / 4}$ such rectangles, we see that $\left\|E f_{R}\right\|_{L^{q}(Y)} \gtrsim R^{(-1 / 2)+(5 /(4 q))}$. So the exponent $q$ in (7) must satisfy $(-1 / 2)+(5 /(4 q)) \leq-1 / 4$, and so a necessary condition for (7) is $q \geq 5$, which is again far from the sufficient condition $q \geq 6$ guaranteed by (2).

The results of this paper will show that (6) and (7) indeed hold for $q>3$ and $q>5$, respectively. As it turns out, we can establish these sharp (up to the endpoints $q=3$ and $q=5$ ) estimates on $X$ and $Y$ as follows.

We first prove a weighted restriction estimate

$$
\|E f\|_{L^{q_{1}}(H d x)} \lesssim A_{\beta}(H)^{1 / q_{1}}\|f\|_{L^{2}(S)}
$$

that holds whenever $0<\beta<1 / 2$ and $q_{1}>2$, and then combine it with the weighted Hölder-type inequality of Theorem 1.1 to conclude that (6) holds for $q>3$. In doing so, we realize that the same argument shows that the estimate

$$
\|E f\|_{L^{q_{2}}(H d x)} \lesssim A_{\alpha}(H)^{1 / q_{2}}\|f\|_{L^{2}(S)}
$$

holds whenever $1 / 2 \leq \alpha \leq 1$ and $q_{2}>4 \alpha$. Combining the last estimate with a corollary (see Corollary 4-A ) of the fractal restriction theorem of Du and Zhang [5], we see that (7) holds for $q>5$. For more details, we refer the reader to Theorem 2.1 and Subsection 3.4.

The strategy that we explore in this paper of proving restriction estimates on specific sets, such as the sets $X$ and $Y$ in the above examples, by first proving restriction estimates for all weights $H$ of low fractal dimensions and then upgrading the estimates to higher fractal dimensions is reminiscent of the polynomial method of [9] and [10] that upgrades restriction estimates from low algebraic dimensions to higher ones.

When it comes to weighted $L^{\infty}$-based estimates, i.e. $L^{\infty}(S) \rightarrow L^{q}(H d x)$ estimates, the situation becomes much harder, and we will postpone that discussion to the next section. 


\section{Results And methodology}

Any restriction estimate $\|E f\|_{L^{q}\left(\mathbb{R}^{n}\right)} \lesssim\|f\|_{L^{p}(S)}$ is equivalent to the weighted estimate $\|E f\|_{L^{q}(H d x)} \lesssim A_{n}(H)^{1 / q}\|f\|_{L^{p}(S)}$. In fact, taking $H=1$, we see that the latter estimate implies the former. On the other hand, since the surface $S$ is compact, we can find a $C_{0}^{\infty}$ function $\phi$ on $\mathbb{R}^{n}$ that satisfies $|\phi| \geq 1$ on $S$ and $\widehat{\phi}$ is compactly supported. Given $f \in L^{p}(S)$, we define $g \in L^{p}(S)$ by $g=f / \phi$, and we observe that $|g| \leq|f|, E f=(E g) * \widehat{\phi}$, and $|E f|^{q} \lesssim|E g|^{q} *|\widehat{\phi}|$. The non-weighted estimate applied to $g$ then tells us that

$$
\begin{aligned}
\int|E f(x)|^{q} H(x) d x & \lesssim \int|E g(y)|^{q} \int|\widehat{\phi}(x-y)| H(x) d x d y \\
& \lesssim A_{n}(H) \int|E g(y)|^{q} d y \lesssim A_{n}(H)\|g\|_{L^{p}(S)}^{q} \leq A_{n}(H)\|f\|_{L^{p}(S)}^{q}
\end{aligned}
$$

In particular, the Tomas-Stein estimate (2) has the following weighted version:

$$
\int|E f(x)|^{q} H(x) d x \lesssim A_{\alpha}(H)\|f\|_{L^{2}(S)}^{q}
$$

for $0<\alpha \leq n$ and $q \geq(2 n+2) /(n-1)$, where we have used the fact that $A_{n}(H) \leq A_{\alpha}(H)$.

Remark 2.1. For establishing (2) (and hence (8)), the assumption requiring the surface $S$ to have a strictly positive second fundamental can be relaxed to just requiring $S$ to have a nowhere vanishing Gaussian curvature.

With the restriction conjecture being open, it is therefore natural to investigate the situation when $\alpha<n$. This has been the subject of two recent papers 4 and 17. Both papers employed Guth's polynomial partitioning method from [9] and [10].

We would like to mention at this point that weighted estimates of the form

$$
\int_{B(0, R)}|E f(x)|^{q} H(x) d x \leq C A_{\alpha}(H) R^{\beta}\|f\|_{L^{2}(S)}^{q} \quad(R \geq 1),
$$

where we integrate over the ball $B(0, R)$ instead of $\mathbb{R}^{n}$, and allow a positive power of the radius $R$ on the right-hand side of the estimate, have been studied extensively in the literature due to their important applications in studying decay properties of Fourier transforms of measures and, consequently, Falconer's conjecture concerning distance sets in geometric measure theory. For such results, we refer the reader to [15], 22], 16], 6], 7], [14, [17, 4], 5], [12] and the references contained within these papers. The results of the present paper do not lead to any progress in the direction of Falconer's conjecture. In fact, and as we mentioned in the paper's abstract and introduction, our main concern is to study the restriction problem on subsets $X$ of $\mathbb{R}^{n}$ with Lebesgue measure $|X|=\infty$ and satisfying the dimentionality property: $\left|X \cap B_{R}\right| \lesssim R^{\alpha}$ for all balls $B_{R}$ in $\mathbb{R}^{n}$ of radius $R \geq 1$. We will, however, use known facts about the decay properties of the spherical means of Fourier transforms of measures to establish some of the lower bounds in Theorems 2.2 and 2.3 .

As the title of the present paper indicates, we are here mostly interested in studying the restriction problem in low fractal dimensions. In fact, this paper proves new weighted $L^{2}$-based restriction estimates, i.e. $L^{2}(S) \rightarrow L^{q}(H d x)$ estimates, in $\mathbb{R}^{n}, n \geq 3$, for $0<\alpha \leq(n+1) / 2$ (see Theorem 2.1). In particular, if $X$ is as in the 
previous paragraph, then, taking $H$ to be the characteristic function of $X$, we get new $L^{2}(S) \rightarrow L^{q}(X)$ restriction estimates.

In the plane, we prove new weighted $L^{2}$-based restriction estimates in the full range $0<\alpha<2$ of fractal dimensions. This is one important aspect of the approach we follow, because the results of [4] and [17] do not include the plane.

In the regime $0<\alpha \leq n / 2$, the best known weighted $L^{2}$-based restriction estimates were obtained in [17] for $n=3$, and in [4] for $n \geq 3$.

The authors of [4] proved that in $\mathbb{R}^{n}, n \geq 3$, to every $\epsilon>0$ there is a constant $C_{\epsilon}$ such that

$$
\int_{B(0, R)}|E f(x)|^{2 n /(n-1)} H(x) d x \leq C_{\epsilon} R^{\epsilon} A_{\alpha}(H)\|f\|_{L^{2}(S)}^{2 n /(n-1)}
$$

whenever $f \in L^{2}(S), 0<\alpha \leq \max [n / 2,2], H$ is a weight of dimension $\alpha$, and $R \geq 1$. (See [4, Theorem 1.8 and Remark 1.10].) In (9), the constant $C_{\epsilon}$ is only allowed to depend on $\epsilon, \alpha, n$, and $S$. Estimates such as (9), where one integrates $E f$ over a ball of radius $R$ instead of the entire $\mathbb{R}^{n}$, are often referred to in the literature as local restriction estimates. Also, to emphasize the fact that the function $E f$ is being integrated over $\mathbb{R}^{n}$, estimates such as (3) and (8) are often called global restriction estimates.

In [17], (9) was proved in $\mathbb{R}^{3}$, but only for $0<\alpha \leq 3 / 2$ and with $A_{\alpha}(H)$ replaced by $\max \left[A_{\alpha}(H), A_{\alpha}(H)^{1 / 4}\right]$.

Remark 2.2. In [4, weights were defined in a slightly different way than in this paper. For $0<\alpha \leq n$, the authors of 4 ] denoted by $\mathcal{F}_{\alpha, n}$ the set of all non-negative measurable functions $H$ on $\mathbb{R}^{n}$ that satisfy $\int_{B\left(x_{0}, R\right)} H(x) d x \leq R^{\alpha}$ for all $x_{0} \in \mathbb{R}^{n}$ and $R \geq 1$, and wrote (9) as:

$$
\int_{B(0, R)}|E f(x)|^{2 n /(n-1)} H(x) d x \leq C_{\epsilon}\|f\|_{L^{2}(S)}^{2 n /(n-1)}
$$

whenever $f \in L^{2}(S), 0<\alpha \leq n / 2, H \in \mathcal{F}_{\alpha, n}$, and $R \geq 1$. The estimates (91) and (10) are equivalent. Clearly, (10) implies (9). To establish the reverse implication, given $H \in \mathcal{F}_{\alpha, n}$ and $N \in \mathbb{N}$, we let $H_{N}=N^{-1} \chi_{\left\{x \in \mathbb{R}^{n}: H(x) \leq N\right\}} H$, observe that $A_{\alpha}\left(H_{N}\right) \leq N^{-1}$, apply (9) with $H_{N}$, send $N$ to infinity, and arrive at (10) via the monotone convergence theorem.

The polynomial method for proving restriction estimates that was developed in 9] and [10] in the non-weighted setting, and adapted in [4] and [17] to the weighted setting, cannot prove restriction estimates for exponents $q<2 n /(n-1)$. In fact, the polynomial method has a key induction argument in the non-algebraic (or cellular) case in which the condition $q \geq 2 n /(n-1)$ is crucial for closing the induction. Since one naturally expects $q$ to go below $2 n /(n-1)$ as $\alpha$ becomes smaller (and, as one learns from Theorem 2.1 turns out to indeed be the case), the polynomial method does not appear to be of much help in handling the $0<\alpha<n / 2$ case.

Also, the polynomial method proves local restriction estimates. In the nonweighted setting, this is not a serious limitation, because one can turn local restriction estimates into global ones by using Tao's $\epsilon$-removal lemma from [19. In the weighted setting, however, the $\epsilon$-removal lemma can only be applied in some special cases (see [17, Section 2]).

In this paper, we prove global weighted $L^{2}$-based restriction estimates, and we manage to go below the $2 n /(n-1)$ threshold (when $0<\alpha<n / 2)$ as follows. We 
divide $0<\alpha \leq n$ into three regimes: $0<\alpha<(n-1) / 2,(n-1) / 2 \leq \alpha \leq n / 2$, and $n / 2<\alpha \leq n$.

In the first regime, we prove an $L^{2}(S) \rightarrow L^{q}(H d x)$ restriction estimate that holds for all $q>2$, and which is sharp up to the endpoint $q=2$. In this part of the proof, we use ideas from Bourgain's paper [1] to utilize the decay we have on the Fourier transform of the surface measure $\sigma$ on $S$.

In the second regime, we prove an $L^{2}(S) \rightarrow L^{q}(H d x)$ restriction estimate that holds for all $q>4 \alpha /(n-1)$. To obtain this result, we combine the result that we have obtained in the first regime with a corollary of Theorem 1.1 (see Corollary 2.1 below).

Once we have established our restriction estimates in the regime $(n-1) / 2 \leq$ $\alpha \leq n / 2$ via Corollary 2.1, we combine them with the fractal restriction theorem of Du and Zhang [5] to obtain new $L^{2}(S) \rightarrow L^{q}(H d x)$ estimates in the regime $n / 2<\alpha \leq(n+1) / 2$ for $n \geq 3$, and $n / 2<\alpha<n$ for $n=2$. As will become apparent during the proof of Theorem 2.1] in the plane we will able to use the full strength of the theorem of Du and Zhang, but in $\mathbb{R}^{n}, n \geq 3$, we will need to weaken the Du-Zhang theorem before we can combine it with the estimates from the second regime.

In the opposite direction, it turns out that our $L^{2}$-based estimates actually improve on the fractal restriction theorem of [5] when $0<\alpha<n / 2$ (see Corollary 4.1).

Here are the main results of this paper concerning $L^{2}$-based estimates.

Theorem 2.1. Suppose $n \geq 2$ and $S$ is a smooth compact hypersurface in $\mathbb{R}^{n}$ with a strictly positive second fundamental form. Then

$$
\int|E f(x)|^{q} H(x) d x \lesssim A_{\alpha}(H)\|f\|_{L^{2}(S)}^{q}
$$

for all functions $f \in L^{2}(S)$ and weights $H$ on $\mathbb{R}^{n}$ of dimension $\alpha$ whenever

$$
q> \begin{cases}2 & \text { if } 0<\alpha<(n-1) / 2 \\ 4 \alpha /(n-1) & \text { if }(n-1) / 2 \leq \alpha \leq n / 2 \\ 2 \alpha+2 & \text { if } n=2 \text { and } 1<\alpha<2 \\ (2 n /(n-1))+2-(n / \alpha) & \text { if } n \geq 3 \text { and } n / 2<\alpha \leq(n+1) / 2\end{cases}
$$

We remark to the reader that the $q>(2 n /(n-1))+2-(n / \alpha)$ result of Theorem 2.1 is in fact true for $(n-1) / 2 \leq \alpha \leq n$, but in the regime $(n+1) / 2<\alpha<n$ it becomes inferior to the estimate in Proposition 6.2 that we state and prove in Section 6 below, and at $\alpha=n$ it becomes inferior to the Tomas-Stein estimate (8).

The reason for not stating Proposition 6.2 here is due to the fact its proof does not follow the strategy outlined above. Instead, the proof of Proposition 6.2 combines the Du-Zhang estimate from [5] with the method that Bourgain developed in [1] to upgrade local restriction estimates to global ones.

The assumption that the surface $S$ has a strictly positive second fundamental form is only needed for the $q>2 \alpha+2$ and $q>(2 n /(n-1))+2-(n / \alpha)$ results of Theorem 2.1 (because of the need to use the fractal restriction theorem of [5]). For the other two results, we only need $S$ to have a nowhere vanishing Gaussian curvature, as will become clear during the proof of Theorem 2.1 (see also Remark 2.1 and Proposition 6.1). 
The ranges of the exponent $q$ in Theorem 2.1 are all sharp (up to the endpoints) in $\mathbb{R}^{2}$. In $\mathbb{R}^{n}, n \geq 3$, we are only able to show that the $q>2$ range is sharp (again up to the endpoint). These results are detailed in the following theorem.

Theorem 2.2. Let $S$ be the unit sphere in $\mathbb{R}^{n}$. Suppose that to every $\epsilon>0$ there is a constant $C_{\epsilon}$ such that

$$
\int_{B(0, R)}|E f(x)|^{q} H(x) d x \leq C_{\epsilon} R^{\epsilon} A_{\alpha}(H)\|f\|_{L^{2}(S)}^{q}
$$

for all functions $f \in L^{2}(S)$, weights $H$ on $\mathbb{R}^{n}$ of dimension $\alpha$, and radii $R \geq 1$. Then

$$
q \geq \begin{cases}2 & \text { if } n \geq 3 \text { and } 0<\alpha \leq n-2 \\ (2 \alpha+2) /(n-1) & \text { if } n \geq 2 \text { and } n-2<\alpha \leq n \\ 2 & \text { if } n=2 \text { and } 0<\alpha<1 / 2 \\ 4 \alpha & \text { if } n=2 \text { and } 1 / 2 \leq \alpha \leq 1\end{cases}
$$

Before starting the discussion of weighted $L^{\infty}$-based estimates, we make a couple of definitions and state a corollary of Theorem 1.1.

For $0<\alpha \leq n$ and $1 \leq p \leq \infty$, we define $Q(\alpha, p)$ to be the infimum of all numbers $q>0$ such that the following holds: there is a constant $C$ such that

$$
\int|E f(x)|^{q} H(x) d x \leq C A_{\alpha}(H)\|f\|_{L^{p}(S)}^{q}
$$

for all functions $f \in L^{p}(S)$ and weights $H$ on $\mathbb{R}^{n}$ of dimension $\alpha$. The constant $C$ is allowed to depend on $n, \alpha, p$, and $q$; but, of course, not on $f$ or $H$.

We also define $Q_{\text {loc }}(\alpha, p)$ to be the infimum of all numbers $q>0$ such that the following holds: to every $\epsilon>0$ there is a constant $C_{\epsilon}$ such that

$$
\int_{B(0, R)}|E f(x)|^{q} H(x) d x \leq C_{\epsilon} R^{\epsilon} A_{\alpha}(H)\|f\|_{L^{p}(S)}^{q}
$$

for all functions $f \in L^{p}(S)$, weights $H$ on $\mathbb{R}^{n}$ of dimension $\alpha$, and radii $R \geq 1$. The constant $C_{\epsilon}$ is allowed to depend on $\epsilon, n, \alpha, p$, and $q$.

For applications in the Fourier restriction context, it will be convenient to state the following corollary of Theorem 1.1 .

Corollary 2.1. Suppose $n \geq 2$ and $0<\beta<\alpha \leq n$. Then

$$
\frac{Q(\alpha, p)}{\alpha} \leq \frac{Q(\beta, p)}{\beta} \quad \text { and } \quad \frac{Q_{\mathrm{loc}}(\alpha, p)}{\alpha} \leq \frac{Q_{\mathrm{loc}}(\beta, p)}{\beta} \text {. }
$$

In view of the fact that Corollary 2.1 holds for all $1 \leq p \leq \infty$, the strategy we outlined above for deriving restriction estimates by breaking $0<\alpha \leq n$ into different regimes works as well for $L^{\infty}$-based estimates as it did for $L^{2}$-based estimates. But, unlike the $L^{2}$-based situation, we are unable to prove a favorable $L^{\infty}$-based estimate for small $\alpha$. In fact, establishing a local $L^{\infty}(S) \rightarrow L^{1}\left(\chi_{B(0, R)} H d x\right)$ restriction estimate for $0<\alpha<(n-1) / 2$ would imply (via Corollary 2.1) a local $L^{\infty}(S) \rightarrow L^{2 n /(n-1)}(B(0, R))$ estimate, which would essentially solve the restriction problem in $\mathbb{R}^{n}$. So it becomes natural to investigate if such an estimate is feasible. For example, could $Q_{\text {loc }}(\alpha, \infty) \rightarrow 0$ as $\alpha \rightarrow 0$ ? The next theorem tells us that $Q_{\text {loc }}(\alpha, \infty) \geq(n-1) / n$ for small $\alpha$, but proving this lower bound turned out to be much harder than the author had initially expected. 
Theorem 2.3. Let $S$ be the unit sphere in $\mathbb{R}^{n}$. Then

$$
Q_{\mathrm{loc}}(\alpha, \infty) \geq \begin{cases}(n-1) / n & \text { if } n \geq 3 \text { and } 0<\alpha \leq(n-1)^{2} /(2 n), \\ 2 \alpha /(n-1) & \text { if } n \geq 3 \text { and }(n-1)^{2} /(2 n) \leq \alpha \leq n, \\ 1 / 2 & \text { if } n=2 \text { and } 0<\alpha \leq 1 / 6 \\ 3 \alpha & \text { if } n=2 \text { and } 1 / 6 \leq \alpha \leq 1 \\ \alpha+2 & \text { if } n=2 \text { and } 1 \leq \alpha \leq 2\end{cases}
$$

The proofs of the first and last inequalities in Theorem 2.3 (as well as the first inequality in Theorem 2.2) involve some geometric measure theory. In particular, the last inequality depends on a theorem of Bennett and Vargas [2] about the decay of the $L^{1}$ circular means of Fourier transforms of measures.

The rest of the paper is organized as follows. In the next section, we give some interesting examples. In Section 4, we discuss the fractal restriction theorem of $\mathrm{Du}$ and Zhang [5] and show how Theorem 2.1 improves on it when $0<\alpha<n / 2$. Section 5 is dedicated to the proofs of Theorem 1.1 and Corollary 2.1. Section 6 proves estimates in the regimes $0<\alpha<(n-1) / 2$ and $(n+1) / 2<\alpha<n$. The last four sections of the paper are the proofs of Theorems [2.1, 2.2, and 2.3.

\section{EXAMPLES}

3.1. Restriction estimates in neighborhoods of algebraic varieties. Let $n \geq$ $2, Z$ be a real algebraic variety in $\mathbb{R}^{n}$ of dimension $k$ that is defined by polynomials of degree at most $D$, and $N_{\rho}(Z)$ be the $\rho$-neighborhood of $Z$. Then a theorem of Wongkew [21] tells us that

$$
\left|N_{\rho}(Z) \cap B_{R}\right| \leq C_{n}(D \rho)^{n-k} R^{k}
$$

for any ball $B_{R} \subset \mathbb{R}^{n}$ of radius $R>0$, where $C_{n}$ is a constant that depends only on $n$. This inequality implies that the characteristic function of $N_{\rho}(Z)$ is a weight on $\mathbb{R}^{n}$ of dimension $k$. Moreover, $A_{k}\left(\chi_{N_{\rho}(Z)}\right) \leq C_{n}(D \rho)^{n-k}$. Therefore, if $n \geq 3$ and $1 \leq k \leq(n+1) / 2$, then Theorem [2.1] applies and tells us that

$$
\int_{N_{\rho}(Z)}|E f(x)|^{q} d x \lesssim(D \rho)^{n-k}\|f\|_{L^{2}(S)}^{q}
$$

for all $f \in L^{2}(S)$ whenever

$$
q> \begin{cases}\max [2,4 k /(n-1)] & \text { if } 1 \leq k \leq n / 2 \\ (2 n /(n-1))+2-(n / k) & \text { if } n / 2<k \leq(n+1) / 2 .\end{cases}
$$

Furthermore, if $n=2$ and $Z$ is the zero set of a polynomial $P$ in two real variables of degree $D \geq 1$, then Theorem 2.1 gives the estimate

$$
\int_{N_{\rho}(Z)}|E f(x)|^{q} d x \lesssim D \rho\|f\|_{L^{2}(S)}^{q} \quad(q>4) .
$$

We also refer the reader to the example at the end of Section 6 for a similar estimate in higher dimensions.

3.2. An example of the $\alpha=n / 2$ case. As a second example, we consider the set $\Omega \subset \mathbb{R}^{n}$ given by

$$
\Omega=\left\{x=\left(\bar{x}, x_{n}\right) \in \mathbb{R}^{n-1} \times \mathbb{R}:\left|x_{n}\right| \leq|\bar{x}|^{1-(n / 2)}\right\} .
$$


It is easy to see that the characteristic function of $\Omega$ is a weight on $\mathbb{R}^{n}$ of dimension $n / 2$ and with $A_{n / 2}\left(\chi_{\Omega}\right) \lesssim 1$. In fact, if $\bar{x}_{0} \in \mathbb{R}^{n-1}$ and $R \geq 1$, then

$$
\int_{B\left(\bar{x}_{0}, R\right)}|\bar{x}|^{1-(n / 2)} d \bar{x} \leq \begin{cases}\int_{B(0,11 R)}|\bar{x}|^{1-(n / 2)} d \bar{x} \lesssim R^{n / 2} & \text { if }\left|\bar{x}_{0}\right| \leq 10 R, \\ \left(\left|\bar{x}_{0}\right|-R\right)^{1-(n / 2)} \int_{B\left(\bar{x}_{0}, R\right)} d x \lesssim R^{n / 2} & \text { if }\left|\bar{x}_{0}\right|>10 R .\end{cases}
$$

Therefore, (9) gives us the local estimate

$$
\int_{\Omega \cap B_{R}}|E f(x)|^{2 n /(n-1)} d x \lesssim R^{\epsilon}\|f\|_{L^{2}(S)}^{2 n /(n-1)},
$$

whereas Theorem 2.1 gives us the global estimate

$$
\int_{\Omega}|E f(x)|^{q} d x \lesssim\|f\|_{L^{2}(S)}^{q} \quad(q>2 n /(n-1)) .
$$

(See Remark 3.1 following the next example.)

3.3. An example in $\mathbb{R}^{3}$. Our third example, which takes place in $\mathbb{R}^{3}$, needs the following result from [4]:

$$
\int_{B_{R}}|E f(x)|^{3} H(x) d x \leq C_{\epsilon} R^{\epsilon} A_{\alpha}(H)\|f\|_{L^{2}(S)}^{3}
$$

for all functions $f \in L^{2}(S)$ and weights $H$ on $\mathbb{R}^{3}$ of dimension $3 / 2 \leq \alpha \leq 2$. This local estimate implies that $Q_{\text {loc }}(2,2) \leq 3$, and so Corollary 2.1 (applied with $\beta=2$ and $n=3$ ) implies that

$$
Q_{\text {loc }}(\alpha, 2) \leq(3 / 2) \alpha \quad(2<\alpha \leq(11-\sqrt{13}) / 3) .
$$

(Proposition 6.2 gives a better result when $(11-\sqrt{13}) / 3<\alpha<3$, and so does (8) when $\alpha=3$.)

Now, for $0<b \leq 1$, we define

$$
\Omega_{b}=\cup_{l=1}^{\infty} \mathbb{R}^{2} \times\left[l^{1 / b}, 1+l^{1 / b}\right]
$$

and we observe that the characteristic function of $\Omega_{b}$ is a weight on $\mathbb{R}^{3}$ of dimension $2+b$ and with $A_{2+b}\left(\chi_{\Omega_{b}}\right) \lesssim 1$. So (13) tells us that

$$
\int_{\Omega_{b} \cap B_{R}}|E f(x)|^{q} d x \lesssim R^{\epsilon}\|f\|_{L^{2}(S)}^{q}
$$

for all $f \in L^{2}(S)$ whenever $b \leq(5-\sqrt{13}) / 3$ and $q \geq(3 / 2)(2+b)$. Combining this local estimate with the $\epsilon$-removal argument of [17, Corollary 2.1] (which is a small modification on Tao's $\epsilon$-removal lemma from [19] that works for some special weights such as the characteristic function of $\Omega_{b}$ ), we conclude that the global estimate

$$
\int_{\Omega_{b}}|E f(x)|^{q} d x \lesssim\|f\|_{L^{2}(S)}^{q}
$$

holds whenever $b \leq(5-\sqrt{13}) / 3$ and $q>(3 / 2)(2+b)$.

Remark 3.1. The $\epsilon$-removal argument of [17, Corollary 2.1] does not apply when $H$ is the characteristic function of the set $\Omega$ in the second example (Subsection 3.2), so the author is not sure whether (12) can be derived from (111) when $n \geq 3$. 
3.4. The two examples from the Introduction - revisited. For $0 \leq b \leq 1$, we define

$$
X_{b}=\left\{(x, y) \in \mathbb{R}^{2}: x>0 \text { and } 0 \leq y \leq x^{-b}\right\} .
$$

If $b<1$, then the characteristic function of $X_{b}$ is a weight on $\mathbb{R}^{2}$ of dimension $1-b$, and $A_{1-b}\left(\chi_{X_{b}}\right) \lesssim 1$. If $b=1$, then $\chi_{X_{b}}$ is a weight on $\mathbb{R}^{2}$ of dimension $\alpha$ and $A_{\alpha}\left(\chi_{X_{b}}\right) \lesssim 1$ for all $0<\alpha \leq 2$. Therefore, by Theorem 2.1, we have

$$
\int_{X_{b}}|E f(x)|^{q} d x \lesssim\|f\|_{L^{2}(S)}^{q} \quad(q>\max [2,4(1-b)]) .
$$

When $b=1$, we also have

$$
\int_{X_{1} \cap B_{R}}|E f(x)|^{q} d x \lesssim(\log R)\|f\|_{L^{2}(S)}^{q} \quad(q>0, R \geq 1),
$$

but we do not have an $\epsilon$-removal theorem that would turn this local estimate into a global one.

We saw in the Introduction that the range of $q$ in (14) is sharp (up to the end point $q=4(1-b)$ ) when $b=1 / 4$. We will see during the proof of Theorem 2.2 that this range of $q$ is actually sharp for all $0 \leq b \leq 1 / 2$.

Our last example also takes place in the plane. For $0<b<1$, we define

$$
Y_{b}=\cup_{l=1}^{\infty} \mathbb{R} \times\left[l^{1 / b}, 1+l^{1 / b}\right] .
$$

Then the characteristic function of $Y_{b}$ is a weight on $\mathbb{R}^{2}$ of dimension $1+b$ and with $A_{1+b}\left(\chi_{Y_{b}}\right) \lesssim 1$. So Theorem 2.1 gives the estimate

$$
\int_{Y_{b}}|E f(x)|^{q} d x \lesssim\|f\|_{L^{2}(S)}^{q} \quad(q>4+2 b) .
$$

We saw in the Introduction that the range of $q$ in (15) is sharp (up to the endpoint $q=4+2 b$ ) when $b=1 / 2$. We will see during the proof of Theorem 2.2 that this range of $q$ is actually sharp for all $0<b<1$.

\section{On a fractal Restriction theOREm of Du AND Zhang}

Throughout this section, we denote a cube in $\mathbb{R}^{n}$ of center $x$ and side-length $r$ by $\widetilde{B}(x, r)$.

Let $\mathcal{P}=\left\{\xi \in \mathbb{R}^{n}: \xi_{n}=\xi_{1}^{2}+\ldots+\xi_{n-1}^{2} \leq 1\right\}$ be the unit paraboloid in $\mathbb{R}^{n}$, and $E_{\mathcal{P}}$ the extension operator associated with $\mathcal{P}$. In a recent paper [5], the following interesting theorem was proved.

Theorem 4-A ([5, Corollary 1.6]). Suppose $n \geq 2,1 \leq \alpha \leq n, R \geq 1, X=\cup_{k} \widetilde{B}_{k}$ is a union of lattice unit cubes in $\widetilde{B}(0, R) \subset \mathbb{R}^{n}$, and

$$
\gamma=\sup \frac{\#\left\{\widetilde{B}_{k}: \widetilde{B}_{k} \subset \widetilde{B}\left(x^{\prime}, r\right)\right\}}{r^{\alpha}},
$$

where the sup is taken over all pairs $\left(x^{\prime}, r\right) \in \mathbb{R}^{n} \times[1, \infty)$ satisfying $\widetilde{B}\left(x^{\prime}, r\right) \subset$ $\widetilde{B}(0, R)$. Then to every $\epsilon>0$ there is a constant $C_{\epsilon}$ such that

$$
\left\|E_{\mathcal{P}} f\right\|_{L^{2}(X)} \leq C_{\epsilon} R^{\epsilon} \gamma^{1 / n} R^{\alpha /(2 n)}\|f\|_{L^{2}(\mathcal{P})}
$$

for all $f \in L^{2}(\mathcal{P})$. 
Theorem 4-A is of interest to us in two ways.

First, Theorem 2.1 of this paper allows us to improve the exponent of $R$ in Theorem 4-A from $\alpha /(2 n)$ to 0 for $0<\alpha \leq(n-1) / 2$, and to $(\alpha / 2)-((n-1) / 4)$ for $(n-1) / 2<\alpha<n / 2$. (We note to the reader that Theorem $4-A$ is a corollary of the main theorem in [5] (see [5. Theorem 1.3]); our results do not appear to improve on the main theorem.)

Second, Theorem 4-A has a corollary that will help us to prove Theorem 2.1 in the regime $n / 2<\alpha \leq(n+1) / 2$, as well as Proposition 6.2.

We start by proving the corollary to Theorem 2.1 that improves on Theorem $4-\mathrm{A}$ when $0<\alpha<n / 2$.

Corollary 4.1. Suppose $n, R, X$, and $\gamma$ are as in Theorem 4-A. Also, suppose that $S$ is a smooth compact hypersurface in $\mathbb{R}^{n}$ with a nowhere vanishing Gaussian curvature, and $E=E_{S}$ is the extension operator on $S$. Then to every $\epsilon>0$ there is a constant $C_{\epsilon}$ such that

$$
\|E f\|_{L^{2}(X)} \leq C_{\epsilon} R^{\epsilon} \gamma^{1 / 2} R^{e(\alpha)}\|f\|_{L^{2}(\mathcal{S})}
$$

for all $f \in L^{2}(\mathcal{S})$, where

$$
e(\alpha)= \begin{cases}0 & \text { if } 0<\alpha \leq(n-1) / 2, \\ (\alpha / 2)-((n-1) / 4) & \text { if }(n-1) / 2<\alpha \leq n / 2 .\end{cases}
$$

Proof. Theorem 2.1 provides us with the local estimate

$$
\int_{B(0, R)}|E f(x)|^{q(\alpha)} H(x) d x \leq C_{\epsilon} R^{\epsilon} A_{\alpha}(H)\|f\|_{L^{2}(S)}^{q(\alpha)}
$$

with

$$
q(\alpha)= \begin{cases}2 & \text { if } 0<\alpha \leq(n-1) / 2 \\ 4 \alpha /(n-1) & \text { if }(n-1) / 2<\alpha \leq n / 2 .\end{cases}
$$

We let $H$ be the characteristic function of $X$. By the definition of $\gamma$, we have

$$
\int_{\widetilde{B}\left(x_{0}, r\right)} H(x) d x \leq \gamma(r+2)^{\alpha} \leq \gamma(3 r)^{\alpha}
$$

for all $x_{0} \in \mathbb{R}^{n}$ and $r \geq 1$. Thus $H$ is a weight on $\mathbb{R}^{n}$ of dimension $\alpha$, and $A_{\alpha}(H) \lesssim \gamma$. This immediately proves the corollary for $0<\alpha \leq(n-1) / 2$.

For $(n-1) / 2<\alpha \leq n / 2$, we apply Hölder's inequality, to get

$$
\begin{aligned}
& \int_{X}|E f(x)|^{2} d x=\int_{B(0, R)}|E f(x)|^{2} H(x) d x \\
& \quad \leq\left(C_{\epsilon} R^{\epsilon} A_{\alpha}(H)\|f\|_{L^{2}(S)}^{q(\alpha)}\right)^{2 / q(\alpha)}\left(\int_{B(0, R)} H(x) d x\right)^{1-(2 / q(\alpha))} \\
& \quad \leq\left(C_{\epsilon} R^{\epsilon} A_{\alpha}(H)\|f\|_{L^{2}(S)}^{q(\alpha)}\right)^{2 / q(\alpha)}\left(A_{\alpha}(H) R^{\alpha}\right)^{1-(2 / q(\alpha))} \\
& =\left(C_{\epsilon} R^{\epsilon}\right)^{2 / q(\alpha)} A_{\alpha}(H) R^{2 e(\alpha)}\|f\|_{L^{2}(S)}^{2},
\end{aligned}
$$

as claimed.

Theorem 4-A has the following corollary that will be needed to prove Theorem 2.1 in the regime $n / 2<\alpha \leq(n+1) / 2$, and Proposition 6.2 
Corollary 4-A ([5]). Suppose $n \geq 2,1 \leq \alpha \leq n, S$ is a smooth compact hypersurface in $\mathbb{R}^{n}$ with a strictly positive second fundamental form, and $E=E_{S}$ is the extension operator on $S$. Then to every $\epsilon>0$ there is a constant $C_{\epsilon}$ such that

$$
\int_{B(0, R)}|E f(x)|^{2} H(x) d x \leq C_{\epsilon} R^{\epsilon} A_{\alpha}(H) R^{\alpha / n}\|f\|_{L^{2}(S)}^{2}
$$

for all functions $f \in L^{2}(S)$, weights $H$ on $\mathbb{R}^{n}$ of dimension $\alpha$, and radii $R \geq 1$.

Corollary 4-A is not stated as such in [5], but is very similar to [5, Theorem 2.3]. Also, the proof of [5. Theorem 2.3] is not explicitly given in that paper, because it is very similar to the proof of [5, Theorem 2.2]. Therefore, we will the present the proof of Corollary $4-\mathrm{A}$ here for the reader's convenience.

Proof of Corollary 4-A ([5, Proof of Theorem 2.2]). We may assume that $S$ is the paraboloid $\mathcal{P}$ that was defined at the beginning of this section (see [4, Remark 1.10] and [5, Part (III) of Subsection 2.2] for the justification of this assumption).

We consider a covering $\{\widetilde{B}\}$ of $B(0, R)$ by unit lattice cubes, and for each such cube we define $v(\widetilde{B})=A_{\alpha}(H)^{-1} \int_{\widetilde{B}} H(x) d x$. Also, for $k=0,-1,-2, \ldots$, we set $V_{k}=\left\{\widetilde{B}: 2^{k-1}<n^{-\alpha / 2} v(\widetilde{B}) \leq 2^{k}\right\}$. Since each cube $\widetilde{B}$ is contained in a ball of radius $\sqrt{n}$, we have $v(\widetilde{B}) \leq n^{\alpha / 2}$, so that $\cup_{k} V_{k} \supset \cup \widetilde{B} \supset B(0, R)$.

Let $k_{1}$ be the sup of the set $\left\{k \in \mathbb{Z}: 2^{k} \leq R^{-1000 n}\right\}$. By the pigeonhole principle, there is an integer $k_{0}$ satisfying $k_{1}<k_{0} \leq 0$ such that

$$
\begin{aligned}
& \int_{B(0, R)}|E f(x)|^{2} H(x) d x \\
& \quad \lesssim R^{\epsilon} 2^{k_{0}} A_{\alpha}(H) \sum_{\widetilde{B} \in V_{k_{0}}} \sup _{\widetilde{B}}|E f|^{2}+A_{\alpha}(H)\|f\|_{L^{2}(S)}^{2} O\left(R^{-500 n}\right) .
\end{aligned}
$$

Since the measure $f d \sigma$ is compactly supported and $E f=\widehat{f d \sigma}$, there is a nonnegative rapidly decaying function $\psi$ on $\mathbb{R}^{n}$ such that $\sup _{\widetilde{B}}|E f|^{2} \leq|E f|^{2} * \psi(c(\widetilde{B}))$, where $c(\widetilde{B})$ is the center of $\widetilde{B}$. So

$$
\sup _{\widetilde{B}}|E f|^{2} \lesssim \int_{B\left(c(\widetilde{B}), R^{\epsilon}\right)}|E f(x)|^{2} d x+\|f\|_{L^{2}(S)}^{2} O\left(R^{-1000 n}\right)
$$

and since

$$
\sum_{\widetilde{B} \in V_{k_{0}}} \chi_{B\left(c(\widetilde{B}), R^{\epsilon}\right)} \lesssim R^{n \epsilon}
$$

it follows that

$$
\sum_{\widetilde{B} \in V_{k_{0}}} \sup _{\widetilde{B}}|E f|^{2} \lesssim R^{n \epsilon} \int_{V}|E f(x)|^{2} d x+\|f\|_{L^{2}(S)}^{2} O\left(R^{-500 n}\right),
$$

where $V=\cup_{\widetilde{B} \in V_{k_{0}}} B\left(c(\widetilde{B}), R^{\epsilon}\right)$.

Let $\left\{\widetilde{B}^{*}\right\}$ be the set of all the unit lattice cubes that intersect $V$, and $X=\cup \widetilde{B}^{*}$. Also, let $B_{r}$ be a ball in $\mathbb{R}^{n}$ of radius $r \geq R^{\epsilon}$. We want to estimate the number of the cubes $\widetilde{B}^{*}$ that intersect $B_{r}$. In order to do this, we need to estimate the number of balls $B\left(c(\widetilde{B}), 2 R^{\epsilon}\right)$ that intersect $B_{r}$. 
We have

$$
\int_{B\left(c(\widetilde{B}), R^{\epsilon}\right)} H(x) d x \geq \int_{\widetilde{B}} H(x) d x=v(\widetilde{B}) A_{\alpha}(H) \geq n^{\alpha / 2} 2^{k_{0}-1} A_{\alpha}(H),
$$

so (using (17))

$$
\begin{gathered}
\left(\#\left\{\widetilde{B} \in V_{k_{0}}: B\left(c(\widetilde{B}), 2 R^{\epsilon}\right) \cap B_{r} \neq \emptyset\right\}\right) 2^{k_{0}} A_{\alpha}(H) \\
\lesssim R^{n \epsilon} \int_{B_{3 r}} H(x) d x \leq R^{n \epsilon} A_{\alpha}(H)(3 r)^{\alpha}
\end{gathered}
$$

and so

$$
\#\left\{\widetilde{B} \in V_{k_{0}}: B\left(c(\widetilde{B}), 2 R^{\epsilon}\right) \cap B_{r} \neq \emptyset\right\} \lesssim R^{n \epsilon} 2^{-k_{0}} r^{\alpha} .
$$

Thus

$$
\#\left\{\widetilde{B}^{*}: \widetilde{B}^{*} \subset B_{r}\right\} \lesssim R^{2 n \epsilon} 2^{-k_{0}} r^{\alpha} .
$$

Therefore, we can apply Theorem $4-\mathrm{A}$ with $\gamma \sim R^{2 n \epsilon} 2^{-k_{0}}$ to get

$$
\int_{V}|E f(x)|^{2} d x \leq \int_{X}|E f(x)|^{2} d x \lesssim R^{6 \epsilon}\left(2^{-k_{0}}\right)^{2 / n} R^{\alpha / n}\|f\|_{L^{2}(S)}^{2},
$$

which, combined with (16) and (18), now tells us that

$$
\begin{aligned}
\int_{B(0, R)}|E f(x)|^{2} d x & \lesssim R^{(n+6) \epsilon}\left(2^{k_{0}}\right)^{1-(2 / n)} A_{\alpha}(H) R^{\alpha / n}\|f\|_{L^{2}(S)}^{2} \\
& \lesssim R^{(n+6) \epsilon} A_{\alpha}(H) R^{\alpha / n}\|f\|_{L^{2}(S)}^{2} .
\end{aligned}
$$

We note that for the last inequality, we need the fact $2^{k_{0}}$ is raised to a non-negative power, which is a consequence of the fact that the exponent of $\gamma$ in the estimate of Theorem 4-A is less than or equal to $1 / 2$, which is also the case in the estimate of Corollary 4.1 .

\section{Proof of the weighted Hölder-type inequality and its Corollary}

In this section we prove Theorem 1.1 and Corollary 2.1 .

Proof of Theorem 1.1. For $N \in \mathbb{N}$, we let $\chi_{N}$ be the characteristic function of the set

$$
B(0, N) \cap\left\{x \in \mathbb{R}^{n}: F(x) \leq N\right\},
$$

and we define the function $F_{N}$ by $F_{N}=\chi_{N} F$. Clearly,

$$
\int F_{N}(x) H(x) d x \leq N \int_{B(0, N)} H(x) d x \leq A_{\alpha}(H) N^{1+\alpha}
$$

for all weights $H$ on $\mathbb{R}^{n}$ of dimension $\alpha$. Letting $\beta_{0}=1$ and $C_{0}=N^{1+\alpha}$, this becomes

$$
\int F_{N}(x)^{\beta_{0}} H(x) d x \leq C_{0} A_{\alpha}(H) .
$$

Let $H$ be a weight on $\mathbb{R}^{n}$ of dimension $\alpha$. 
BASSAM SHAYYA

If $p>1$ and $B_{R}$ is a ball in $\mathbb{R}^{n}$ of radius $R \geq 1$, then (19) and Hölder's inequality tell us that

$$
\begin{aligned}
\int_{B_{R}} F_{N}(x)^{\beta_{0} / p} H(x) d x & \leq\left(\int_{B_{R}} F_{N}(x)^{\beta_{0}} H(x) d x\right)^{1 / p}\left(\int_{B_{R}} H(x) d x\right)^{1 / p^{\prime}} \\
& \leq\left(C_{0} A_{\alpha}(H)\right)^{1 / p}\left(A_{\alpha}(H) R^{\alpha}\right)^{1 / p^{\prime}} \\
& =C_{0}^{1 / p} A_{\alpha}(H) R^{\alpha / p^{\prime}}
\end{aligned}
$$

where $p^{\prime}$ is the exponent conjugate to $p$.

We now choose $p$ such that $\alpha / p^{\prime}=\beta$, i.e. $p=\alpha /(\alpha-\beta)$, to conclude that the function $\mathcal{H}(x):=N^{-1 / p} F_{N}(x)^{\beta_{0} / p} H(x)$ is a weight on $\mathbb{R}^{n}$ of dimension $\beta$. Moreover,

$$
A_{\beta}(\mathcal{H}) \leq N^{-1 / p} C_{0}^{1 / p} A_{\alpha}(H) .
$$

Therefore,

$$
\begin{aligned}
\int F_{N}(x)^{\beta} N^{-1 / p} F_{N}(x)^{\beta_{0} / p} H(x) d x & =\int F_{N}(x)^{\beta} \mathcal{H}(x) d x \\
& \leq\left(M_{\beta} F_{N}\right)^{\beta} A_{\beta}(\mathcal{H}) \\
& \leq\left(M_{\beta} F\right)^{\beta} N^{-1 / p} C_{0}^{1 / p} A_{\alpha}(H),
\end{aligned}
$$

where we have used the fact that $F_{N} \leq F$ to conclude that $M_{\beta} F_{N} \leq M_{\beta} F$. Letting $M=\left(M_{\beta} F\right)^{\beta}, \beta_{1}=\beta+\left(\beta_{0} / p\right)$, and $C_{1}=M C_{0}^{1 / p}$, this becomes

$$
\int F_{N}(x)^{\beta_{1}} H(x) d x \leq C_{1} A_{\alpha}(H) .
$$

Iterating the above procedure starting from (20) instead of (19), we arrive at

$$
\int F_{N}(x)^{\beta_{2}} H(x) d x \leq C_{2} A_{\alpha}(H)
$$

with $\beta_{2}=\beta+\left(\beta_{1} / p\right)$ and $C_{2}=M C_{1}^{1 / p}$. Proceeding in this fashion and using mathematical induction, we obtain two sequences $\left\{\beta_{k}\right\}$ and $\left\{C_{k}\right\}$ of non-negative numbers such that

$$
\int F_{N}(x)^{\beta_{k}} H(x) d x \leq C_{k} A_{\alpha}(H),
$$

$\beta_{k}=\beta+\left(\beta_{k-1} / p\right)$, and $C_{k}=M C_{k-1}^{1 / p}$.

Now

$$
\begin{aligned}
& \beta_{k}=\beta+\frac{\beta_{k-1}}{p}=\beta+\left(\beta+\frac{\beta_{k-2}}{p}\right) \frac{1}{p}=\beta+\frac{\beta}{p}+\frac{\beta_{k-2}}{p^{2}}=\beta+\frac{\beta}{p}+\left(\beta+\frac{\beta_{k-3}}{p}\right) \frac{1}{p^{2}} \\
& =\beta+\frac{\beta}{p}+\frac{\beta}{p^{2}}+\frac{\beta_{k-3}}{p^{3}}=\beta+\frac{\beta}{p}+\frac{\beta}{p^{2}}+\cdots+\frac{\beta}{p^{k-1}}+\frac{\beta_{k-k}}{p^{k}}=\beta \frac{1-(1 / p)^{k}}{1-(1 / p)}+\frac{\beta_{0}}{p^{k}}
\end{aligned}
$$

and

$$
\begin{aligned}
& C_{k}=M C_{k-1}^{1 / p}=M\left(M C_{k-2}^{1 / p}\right)^{1 / p}=M^{1+(1 / p)} C_{k-2}^{(1 / p)^{2}} \\
& =M^{1+(1 / p)}\left(M C_{k-3}^{1 / p}\right)^{(1 / p)^{2}}=M^{1+(1 / p)+(1 / p)^{2}} C_{k-3}^{(1 / p)^{3}} \\
& =M^{1+(1 / p)+(1 / p)^{2}+\cdots+(1 / p)^{k-1}} C_{k-k}^{(1 / p)^{k}}=M^{\left(1-(1 / p)^{k}\right) /(1-(1 / p))} C_{0}^{(1 / p)^{k}},
\end{aligned}
$$


so (recalling that $p=\alpha /(\alpha-\beta))$

$$
\lim _{k \rightarrow \infty} \beta_{k}=\frac{\beta}{1-(1 / p)}=\alpha \quad \text { and } \quad \lim _{k \rightarrow \infty} C_{k}=M^{\alpha / \beta}=\left(M_{\beta} F\right)^{\alpha} .
$$

Therefore, letting $k \rightarrow \infty$ in (21) and using Fatou's lemma, we arrive at

$$
\int F_{N}(x)^{\alpha} H(x) d x \leq\left(M_{\beta} F\right)^{\alpha} A_{\alpha}(H) .
$$

Since $F_{N} \rightarrow F$ pointwise on $\mathbb{R}^{n}$ as $N \rightarrow \infty$, a second application of Fatou's lemma gives us

$$
\int F(x)^{\alpha} H(x) d x \leq\left(M_{\beta} F\right)^{\alpha} A_{\alpha}(H) .
$$

Since the last inequality holds for all weights $H$ on $\mathbb{R}^{n}$ of dimension $\alpha$, it follows that $M_{\alpha} F \leq M_{\beta} F$.

Proof of Corollary 2.1, We will only prove the inequality concerning $Q_{\text {loc }}(\alpha, p)$. The proof for $Q(\alpha, p)$ is similar and a little easier.

We may assume $Q_{\text {loc }}(\beta, p)<\infty$ (otherwise, there is nothing to prove). Let $q>Q_{\mathrm{loc}}(\beta, p)$. Then by the definition of $Q_{\mathrm{loc}}(\beta, p)$, to every $\epsilon>0$ there is a constant $C_{\epsilon}$ such that

$$
\int_{B(0, R)}|E f(x)|^{q} \mathcal{H}(x) d x \leq C_{\epsilon} R^{\epsilon} A_{\beta}(\mathcal{H})\|f\|_{L^{p}(S)}^{q}
$$

for all functions $f \in L^{p}(S)$ and weights $\mathcal{H}$ on $\mathbb{R}^{n}$ of dimension $\beta$. Letting $F=$ $\chi_{B(0, R)}|E f|^{q / \beta}$, this implies

$$
M_{\beta}\left(F^{\beta}\right) \leq\left(C_{\epsilon} R^{\epsilon}\|f\|_{L^{p}(S)}^{q}\right)^{1 / \beta} .
$$

Applying Theorem 1.1, we get

$$
M_{\alpha}\left(F^{\beta}\right) \leq\left(C_{\epsilon} R^{\epsilon}\|f\|_{L^{p}(S)}^{q}\right)^{1 / \beta} .
$$

Therefore,

$$
\left(\frac{1}{A_{\alpha}(H)} \int_{B(0, R)}|E f(x)|^{(\alpha / \beta) q} H(x) d x\right)^{1 / \alpha} \leq\left(C_{\epsilon} R^{\epsilon}\|f\|_{L^{p}(S)}^{q}\right)^{1 / \beta}
$$

for all functions $f \in L^{p}(S)$ and weights $H$ on $\mathbb{R}^{n}$ of dimension $\alpha$.

Recalling the definition of $Q_{\text {loc }}(\alpha, p)$, we now have $(\alpha / \beta) q \geq Q_{\text {loc }}(\alpha, p)$. Since this inequality is true for all $q>Q_{\text {loc }}(\beta, p)$, it follows that

$$
(\alpha / \beta) Q_{\mathrm{loc}}(\beta, p) \geq Q_{\mathrm{loc}}(\alpha, p),
$$

as desired.

6. Estimates In the Regimes $0<\alpha<(n-1) / 2$ AND $(n+1) / 2<\alpha<n$

We start by proving two $L^{2}$-based weighted restriction estimates. The first estimate, which is part (i) of Proposition 6.1. proves Theorem 2.1] in the regime $0<\alpha<(n-1) / 2$, and, as discussed in the Introduction, is the base for proving the theorem in the other two regimes $(n-1) / 2 \leq \alpha \leq n / 2$ and $n / 2<\alpha \leq(n+1) / 2$. The second estimate, which is part (ii) of Proposition 6.1, will be one of the main components of the proof of Theorem 2.3. The work we do in this section is based on ideas from [1]. 
BASSAM SHAYYA

Proposition 6.1. Suppose $S$ is a smooth compact hypersurface in $R^{n}$ with a nowhere vanishing Gaussian curvature, and $H$ is a weight on $\mathbb{R}^{n}$ of dimension $0<\alpha<(n-1) / 2$. Then:

(i) To every exponent $q>2$ there is a constant $C_{q}$, which does not depend on $H$, such that

$$
\|E f\|_{L^{q}(H d x)} \leq C_{q} A_{\alpha}(H)^{1 / q}\|f\|_{L^{2}(S)}
$$

for all $f \in L^{2}(S)$.

(ii) To every exponent $q>(n+1-2 \alpha) /(n-2 \alpha)$ there is a constant $\bar{C}_{q}$, which does not depend on $H$, such that

$$
\|E f\|_{L^{q}(H d x)} \leq \bar{C}_{q} A_{\alpha}(H)^{1 /(q(n-2 \alpha))}\|H\|_{L^{2}\left(\mathbb{R}^{n}\right)}^{(n-1-2 \alpha) /(q(n-2 \alpha))}\|f\|_{L^{2}(S)}
$$

for all $f \in L^{2}(S)$.

Proof. We may assume that $H \in L^{1}\left(\mathbb{R}^{n}\right)$. (Otherwise, we multiply $H$ by the characteristic function of the ball $B(0, R)$, obtain an estimate that is uniform in $R$, and then send $R$ to infinity using the fact that $A_{\alpha}\left(\chi_{B(0, R)} H\right) \leq A_{\alpha}(H)$.) We define the measure $\mu$ on $\mathbb{R}^{n}$ by $d \mu=H d x$.

Let $f \in L^{2}(S)$. We need to estimate $\|E f\|_{L^{q}(\mu)}$. We write

$$
\|E f\|_{L^{q}(\mu)}^{q}=\int_{0}^{\|f\|_{L^{1}(S)}} q \lambda^{q-1} \mu(\{|E f| \geq \lambda\}) d \lambda .
$$

The set $\{|E f| \geq \lambda\}$ is contained in

$$
\left\{(\operatorname{Re} E f)_{+} \geq \frac{\lambda}{4}\right\} \cup\left\{(\operatorname{Re} E f)_{-} \geq \frac{\lambda}{4}\right\} \cup\left\{(\operatorname{Im} E f)_{+} \geq \frac{\lambda}{4}\right\} \cup\left\{(\operatorname{Im} E f)_{-} \geq \frac{\lambda}{4}\right\},
$$

where $(\operatorname{Re} E f)_{+}$and $(\operatorname{Re} E f)_{-}$are, respectively, the positive and negative parts of $\operatorname{Re} E f$; and similarly for $\operatorname{Im} E f$. Therefore, it is enough to estimate the $\mu$-measure of the set $\left\{(\operatorname{Re} E f)_{+} \geq \lambda / 4\right\}$. We denote this set by $G$, and we observe that

$$
G=\left\{\operatorname{Re} E f \geq \frac{\lambda}{4}\right\}
$$

for $\lambda>0$. So

$$
\frac{\lambda}{4} \mu(G) \leq \int_{G}(\operatorname{Re} E f) d \mu=\operatorname{Re} \int_{G} E f d \mu=\operatorname{Re} \int \chi_{G} \widehat{f d \sigma} d \mu=\operatorname{Re} \int \widehat{\chi_{G} d \mu} f d \sigma,
$$

and so (by Cauchy-Schwarz)

$$
\lambda^{2} \mu(G)^{2} \leq 16\|f\|_{L^{2}(S)}^{2}\left\|\widehat{\chi_{G} d \mu}\right\|_{L^{2}(S)}^{2} .
$$

By the duality relation of the Fourier transform, we have

$$
\left\|\widehat{\chi_{G} d \mu}\right\|_{L^{2}(S)}^{2}=\int \widehat{\chi_{G} d \mu} \overline{\overline{\chi_{G} d \mu}} d \sigma=\int\left(\overline{\overline{\chi_{G} d \mu}} d \sigma\right) \widehat{\chi_{G}} d \mu=\int\left(\left(\chi_{G} d \mu\right) * \widehat{\sigma}\right) \chi_{G} d \mu \text {, }
$$

$\mathrm{SO}$

$$
\lambda^{2} \mu(G)^{2} \leq 16\|f\|_{L^{2}(S)}^{2} \int\left(\left(\chi_{G} d \mu\right) * \widehat{\sigma}\right) \chi_{G} d \mu .
$$

The next step is to invoke the decay estimate we have on $\widehat{\sigma}:|\widehat{\sigma}(\xi)| \lesssim|\xi|^{-(n-1) / 2}$ for all $|\xi| \geq 1$ (which is a consequence of the nowhere vanishing Gaussian curvature assumption on the surface $S$ ), as well as the dimensionality of the measure $\mu$ :

$$
\mu\left(B\left(x_{0}, R\right)\right)=\int_{B\left(x_{0}, R\right)} H(x) d x \leq A_{\alpha}(H) R^{\alpha}
$$


for all $x_{0} \in \mathbb{R}^{n}$ and $R \geq 1$.

We let $\psi_{0}$ be a $C_{0}^{\infty}$ function on $\mathbb{R}^{n}$ satisfying $0 \leq \psi_{0} \leq 1, \psi_{0}=1$ on $B(0,1)$, and $\psi_{0}=0$ outside $B(0,2)$. Also, for $l \in \mathbb{N}$, we define $\psi_{l}(x)=\psi_{0}\left(x / 2^{l}\right)-\psi_{0}\left(x / 2^{l-1}\right)$. Then $\psi_{l}$ is supported in the ring $2^{l-1} \leq|x| \leq 2^{l+1}$, and

$$
\left(\chi_{G} d \mu\right) * \widehat{\sigma}=\sum_{k=0}^{\infty}\left(\chi_{G} d \mu\right) *\left(\psi_{k} \widehat{\sigma}\right) .
$$

Since $\left|\psi_{0} \widehat{\sigma}\right| \lesssim 1$ and $\left|\psi_{l} \widehat{\sigma}\right| \lesssim 2^{-(l-1)(n-1) / 2}$, we have

$$
\begin{aligned}
\left|\left(\chi_{G} d \mu\right) *\left(\psi_{k} \widehat{\sigma}\right)(x)\right| & \leq \int\left|\psi_{k}(x-y) \widehat{\sigma}(x-y)\right| \chi_{G}(y) d \mu(y) \\
& \lesssim 2^{-k(n-1) / 2} \int \chi_{B\left(x, 2^{k+1}\right)}(y) \chi_{G}(y) d \mu(y) \\
& \lesssim 2^{-k(n-1) / 2} \mu\left(B\left(x, 2^{k+1}\right)\right) \\
& \lesssim A_{\alpha}(H) 2^{-k(n-1-2 \alpha) / 2},
\end{aligned}
$$

and since $\alpha<(n-1) / 2$, it follows that

$$
\left|\left(\chi_{G} d \mu\right) * \widehat{\sigma}(x)\right| \lesssim \sum_{k=0}^{\infty} A_{\alpha}(H) 2^{-k(n-1-2 \alpha) / 2} \lesssim A_{\alpha}(H)
$$

for all $x \in \mathbb{R}^{n}$.

Returning to (23), we now have $\lambda^{2} \mu(G)^{2} \lesssim\|f\|_{L^{2}(S)}^{2} A_{\alpha}(H) \mu(G)$. Therefore, by (22),

$$
\|E f\|_{L^{q}(\mu)}^{q} \lesssim A_{\alpha}(H)\|f\|_{L^{2}(S)}^{2} \int_{0}^{\|f\|_{L^{1}(S)}} \lambda^{q-3} d \lambda \lesssim A_{\alpha}(H)\|f\|_{L^{2}(S)}^{q}
$$

provided $q>2$. This proves part (i).

We note that in proving part (i) we did not use the dimensionality of the measure $\sigma: \sigma\left(B\left(x_{0}, r\right)\right) \lesssim r^{n-1}$ for all $x_{0} \in \mathbb{R}^{n}$ and $r>0$. But the dimensionality of $\sigma$ will be used in proving part (ii) in the following form:

$$
\left\|\widehat{\psi_{k}} * \sigma\right\|_{L^{\infty}} \lesssim 2^{k}
$$

for $k=0,1,2, \ldots$

The inequality (24) is a bound on $\left\|\left(\chi_{G} d \mu\right) *\left(\psi_{k} \widehat{\sigma}\right)\right\|_{L^{\infty}}$, which implies that

$$
\int\left|\left(\chi_{G} d \mu\right) *\left(\psi_{k} \widehat{\sigma}\right)\right| \chi_{G} d \mu \lesssim 2^{-k(n-1-2 \alpha) / 2} A_{\alpha}(H) \mu(G) .
$$

We now derive a second bound on $\int\left|\left(\chi_{G} d \mu\right) *\left(\psi_{k} \widehat{\sigma}\right)\right| \chi_{G} d \mu$. By Plancherel and (25),

$$
\left\|\left(\chi_{G} d \mu\right) *\left(\psi_{k} \widehat{\sigma}\right)\right\|_{L^{2}} \lesssim 2^{k}\left\|\widehat{\chi_{G} H}\right\|_{L^{2}}=2^{k}\left\|\chi_{G} H\right\|_{L^{2}}
$$

so (by Cauchy-Schwarz)

$$
\int\left|\left(\chi_{G} d \mu\right) *\left(\psi_{k} \widehat{\sigma}\right)\right| \chi_{G} d \mu \lesssim 2^{k}\left\|\chi_{G} H\right\|_{L^{2}}^{2} .
$$

Thus

$$
\int\left|\left(\chi_{G} d \mu\right) *\left(\psi_{k} \widehat{\sigma}\right)\right| \chi_{G} d \mu \lesssim \min \left[2^{k}\|H\|_{L^{2}}^{2}, 2^{-k(n-1-2 \alpha) / 2} A_{\alpha}(H) \mu(G)\right]
$$

for $k=0,1,2, \ldots$, where we have used the fact that $\left\|\chi_{G} H\right\|_{L^{2}} \leq\|H\|_{L^{2}}$. 
Returning to (23), we now have

$$
\lambda^{2} \mu(G)^{2} \lesssim\|f\|_{L^{2}(S)}^{2}\left(\sum_{k=0}^{k_{0}} 2^{k}\|H\|_{L^{2}}^{2}+\sum_{k=k_{0}}^{\infty} 2^{-k(n-1-2 \alpha) / 2} A_{\alpha}(H) \mu(G)\right),
$$

where $k_{0}$ is a positive integer that satisfies

$$
2^{k_{0}} \sim\left(\frac{A_{\alpha}(H) \mu(G)}{\|H\|_{L^{2}}^{2}}\right)^{2 /(n+1-2 \alpha)}
$$

Since $(n-1-2 \alpha) / 2>0$, the geometric series converges giving

$$
\lambda^{2} \mu(G)^{2} \lesssim\|f\|_{L^{2}(S)}^{2}\left(A_{\alpha}(H) \mu(G)\right)^{2 /(n+1-2 \alpha)}\|H\|_{L^{2}}^{2(n-1-2 \alpha) /(n+1-2 \alpha)},
$$

which in turn implies that

$$
\mu(G) \lesssim A_{\alpha}(H)^{1 /(n-2 \alpha)}\|H\|_{L^{2}}^{(n-1-2 \alpha) /(n-2 \alpha)}\left(\lambda^{-1}\|f\|_{L^{2}(S)}\right)^{(n+1-2 \alpha) /(n-2 \alpha)} .
$$

Inserting this bound on $\mu(G)$ into (22), we obtain

$$
\begin{aligned}
\|E f\|_{L^{q}(\mu)}^{q} \lesssim & A_{\alpha}(H)^{1 /(n-2 \alpha)}\|H\|_{L^{2}}^{(n-1-2 \alpha) /(n-2 \alpha)}\|f\|_{L^{2}(S)}^{(n+1-2 \alpha) /(n-2 \alpha)} \\
& \times \int_{0}^{\|f\|_{L^{1}(S)}} \lambda^{q-1-(n+1-2 \alpha) /(n-2 \alpha)} d \lambda \\
& \lesssim A_{\alpha}(H)^{1 /(n-2 \alpha)}\|H\|_{L^{2}}^{(n-1-2 \alpha) /(n-2 \alpha)}\|f\|_{L^{2}(S)}^{q}
\end{aligned}
$$

provided $q>(n+1-2 \alpha) /(n-2 \alpha)$, which proves part (ii).

Readers who are familiar with Bourgain's paper [1] will realize that we can follow that paper more closely by inserting a favorable local restriction estimate in the inequality immediately preceding (24). The argument will then proceed as follows.

Suppose $1 \leq \alpha<n$. The last inequality before (24) says

$$
\int \chi_{B\left(x, 2^{k+1}\right)}(y) \chi_{G}(y) d \mu(y) \lesssim \mu\left(B\left(x, 2^{k+1}\right)\right)
$$

We replace this by

$$
\begin{aligned}
\int_{B\left(x, 2^{k+1}\right)} \chi_{G}(y) d \mu(y) & \lesssim \lambda^{-2} \int_{B\left(x, 2^{k+1}\right)}|E f(y)|^{2} H(y) d y \\
& \lesssim \lambda^{-2} 2^{k \epsilon} A_{\alpha}(H) 2^{k \alpha / n}\|f\|_{L^{2}(S)}^{2}
\end{aligned}
$$

where on the first line we used the fact that $\chi_{G} \leq 4 \lambda^{-1}|E f|$, and on the second line we used the Du and Zhang estimate from Corollary 4-A. Inequality (24) becomes

$$
\left|\left(\chi_{G} d \mu\right) *\left(\psi_{k} \widehat{\sigma}\right)(x)\right| \lesssim \lambda^{-2} A_{\alpha}(H) 2^{-k((n-1) / 2-(\alpha / n)-\epsilon)}\|f\|_{L^{2}(S)}^{2},
$$

so that

$$
\int\left|\left(\chi_{G} d \mu\right) *\left(\psi_{k} \widehat{\sigma}\right)\right| \chi_{G} d \mu \lesssim \lambda^{-2} A_{\alpha}(H) 2^{-k((n-1) / 2-(\alpha / n)-\epsilon)}\|f\|_{L^{2}(S)}^{2} \mu(G) .
$$


Combining this inequality with (26), we arrive at

$$
\begin{aligned}
& \int\left|\left(\chi_{G} d \mu\right) *\left(\psi_{k} \widehat{\sigma}\right)\right| \chi_{G} d \mu \\
& \quad \lesssim \min \left[2^{k}\left\|\chi_{G} H\right\|_{L^{2}}^{2}, \lambda^{-2} A_{\alpha}(H) 2^{-k((n-1) / 2-(\alpha / n)-\epsilon)}\|f\|_{L^{2}(S)}^{2} \mu(G)\right] \\
& \quad \leq \mu(G) \min \left[2^{k}, \lambda^{-2} A_{\alpha}(H) 2^{-k((n-1) / 2-(\alpha / n)-\epsilon)}\|f\|_{L^{2}(S)}^{2}\right]
\end{aligned}
$$

for $k=0,1,2, \ldots$, where we have used the fact that $\left\|\chi_{G} H\right\|_{L^{2}}^{2}=\int_{G} H(x)^{2} d x \leq$ $\int_{G} H(x) d x=\mu(G)$.

Returning to (23), we now have

$$
\lambda^{2} \mu(G) \lesssim\|f\|_{L^{2}(S)}^{2}\left(\sum_{k=0}^{k_{0}} 2^{k}+\sum_{k=k_{0}}^{\infty} \lambda^{-2} A_{\alpha}(H) 2^{-k((n-1) / 2-(\alpha / n)-\epsilon)}\|f\|_{L^{2}(S)}^{2}\right),
$$

where $k_{0}$ is a positive integer that satisfies

$$
2^{k_{0}} \sim\left(\lambda^{-2} A_{\alpha}(H)\|f\|_{L^{2}(S)}^{2}\right)^{1 /((n+1) / 2-(\alpha / n)-\epsilon)} .
$$

For the geometric series to converge, we must have $(n-1) / 2-(\alpha / n)-\epsilon>0$, i.e.

$$
\alpha<\frac{n(n-1)}{2}-n \epsilon
$$

This is possible if $\alpha<n(n-1) / 2$. In the plane, this condition becomes $\alpha<1$. But for Corollary 4-A to hold, we need $\alpha \geq 1$, so for the rest of this argument we must work in $\mathbb{R}^{n}$ with $n \geq 3$. So, choosing $\epsilon$ sufficiently small, we get

$$
\lambda^{2} \mu(G) \lesssim\|f\|_{L^{2}(S)}^{2}\left(\lambda^{-2} A_{\alpha}(H)\|f\|_{L^{2}(S)}^{2}\right)^{1 /((n+1) / 2-(\alpha / n)-\epsilon)},
$$

and so

$$
\mu(G) \lesssim \lambda^{-2 q_{\epsilon}}\|f\|_{L^{2}(S)}^{2 q_{\epsilon}} A_{\alpha}(H)^{1 /((n+1) / 2-(\alpha / n)-\epsilon)},
$$

where $q_{\epsilon}=((n+3) / 2-(\alpha / n)-\epsilon) /((n+1) / 2-(\alpha / n)-\epsilon)$.

Inserting the bound we now have on $\mu(G)$ into (22), we obtain

$$
\begin{aligned}
\|E f\|_{L^{q}(\mu)}^{q} & \lesssim\|f\|_{L^{2}(S)}^{2 q_{\epsilon}} A_{\alpha}(H)^{1 /((n+1) / 2-(\alpha / n)-\epsilon)} \int_{0}^{\|f\|_{L^{1}(S)}} \lambda^{q-1-2 q_{\epsilon}} d \lambda \\
& \lesssim A_{\alpha}(H)^{1 /((n+1) / 2-(\alpha / n)-\epsilon)}\|f\|_{L^{2}(S)}^{q}
\end{aligned}
$$

provided $q>2 q_{\epsilon}$. Since

$$
\lim _{\epsilon \rightarrow 0} 2 q_{\epsilon}=2 \frac{n^{2}+3 n-2 \alpha}{n^{2}+n-2 \alpha},
$$

we obtain the following result.

Proposition 6.2. Suppose that $n \geq 3,1 \leq \alpha<n$, and $S$ is a compact $C^{\infty}$ hypersurface in $\mathbb{R}^{n}$ with a strictly positive second fundamental form. Then to every exponent $q>2\left(n^{2}+3 n-2 \alpha\right) /\left(n^{2}+n-2 \alpha\right)$ there is a constant $c_{q}$ satisfying $c_{q}<((n-1) / 2)-(\alpha / n)$ such that the following holds: if $0<\epsilon<c_{q}$, then

$$
\int|E f(x)|^{q} H(x) d x \lesssim A_{\alpha}(H)^{1 /((n+1) / 2-(\alpha / n)-\epsilon)}\|f\|_{L^{2}(S)}^{q}
$$

for all functions $f \in L^{2}(S)$ and weights $H$ on $\mathbb{R}^{n}$ of dimension $\alpha$. 
We note that $2\left(n^{2}+3 n-2 \alpha\right) /\left(n^{2}+n-2 \alpha\right)=2(n+1) /(n-1)$ if $\alpha=n$, so Proposition 6.2 improves on Tomas-Stein for all $1 \leq \alpha<n$. But

$$
2 \frac{n^{2}+3 n-2 \alpha}{n^{2}+n-2 \alpha}>\frac{2 n}{n-1} \quad \text { for } \quad n \geq 3 \text { and } 0<\alpha<\frac{n(n+1)}{2},
$$

so Theorem 2.1 gives a far better result for $0<\alpha \leq n / 2$. In fact, the range of $q$ in Theorem 2.1 is better than that in Proposition 6.2 for $0<\alpha \leq \alpha_{n}$, where $\alpha_{n}$ is the smaller of the two solutions of the equation

$$
2 \frac{n^{2}+3 n-2 \alpha}{n^{2}+n-2 \alpha}=\frac{2 n}{n-1}+2-\frac{n}{\alpha} .
$$

Solving this equation, we see that

$$
\alpha_{n}=\frac{n^{2}+1-\sqrt{n^{4}-4 n^{3}+2 n^{2}+4 n+1}}{4}=\frac{n+1}{2} .
$$

Example. Suppose $n \geq 3$ and $Z$ is the zero set of a polynomial $P$ on $\mathbb{R}^{n}$ of degree $D \geq 1$. Also, suppose $N_{\rho}(Z)$ is the $\rho$-neighborhood of $Z$ and $H$ is the characteristic function of $N_{\rho}(Z)$. As we saw in the first example of Section $3, H$ is a weight on $\mathbb{R}^{n}$ of dimension $n-1$ with $A_{n-1}(H) \leq C_{n} D \rho$. So we can apply Proposition 6.2 with $\alpha=n-1$.

The exponent of $A_{n-1}(H)$ in Proposition 6.2 is

$$
\left(\frac{n+1}{2}-\frac{n-1}{n}-\epsilon\right)^{-1} \leq \frac{2}{n-1}
$$

provided $\epsilon<1 / n$. Therefore, we have the estimate

$$
\int_{N_{\rho}(Z)}|E f(x)|^{q} d x \lesssim(D \rho)^{2 /(n-1)}\|f\|_{L^{2}(S)}^{q} \quad\left(q>2 \frac{n^{2}+n+2}{n^{2}-n+2}\right)
$$

for all $\rho \geq D^{-1}$. One interesting aspect of this estimate is that it holds beyond the $(2 n+2) /(n-1)$ exponent of Tomas-Stein, another interesting aspect is that the exponent of $\rho$ goes to zero as $n \rightarrow \infty$.

\section{Proof of Theorem 2.1}

Having discussed in detail the Du and Zhang fractal restriction theorem, proven the weighted Hölder-type inequality and its corollary, and established a good restriction estimate in fractal dimensions $0<\alpha<(n-1) / 2$, we are now ready to put all those components together and prove Theorem 2.1

Proof of Theorem 2.1. Let $Q(\alpha, 2)$ be the quantity defined right before the statement of Corollary 2.1. We need to show that

$$
Q(\alpha, 2) \leq \begin{cases}2 & \text { if } 0<\alpha<(n-1) / 2, \\ 4 \alpha /(n-1) & \text { if }(n-1) / 2 \leq \alpha \leq n / 2, \\ 2 \alpha+2 & \text { if } n=2 \text { and } 1<\alpha<2, \\ (2 n /(n-1))+2-(n / \alpha) & \text { if } n \geq 3 \text { and } n / 2<\alpha \leq n .\end{cases}
$$

(See the statement of Theorem 2.1 and the paragraph immediately following it.)

Part (i) of Proposition 6.1 immediately gives the inequality on the first line of (27). Then, applying Theorem 2.1 with $0<\beta<(n-1) / 2 \leq \alpha \leq n / 2$, we get

$$
\frac{Q(\alpha, 2)}{\alpha} \leq \frac{Q(\beta, 2)}{\beta} \leq \frac{2}{\beta} \text {. }
$$


Therefore (letting $\beta \rightarrow(n-1) / 2), Q(\alpha, 2) \leq 4 \alpha /(n-1)$.

It remains to prove the last two lines of (27). For this we need Corollary 4-A]

Suppose $n=2$ and $1<\alpha<2$. Also, let $\epsilon>0$ and $f \in L^{2}(S)$. Then Corollary 4-A tells us that

$$
\int_{B_{R}}|E f(x)|^{2} H(x) d x \leq C_{\epsilon} R^{\epsilon} A_{\alpha}(H) R^{\alpha / 2}\|f\|_{L^{2}(S)}^{2}
$$

for all balls $B_{R} \subset \mathbb{R}^{2}$ of radius $R \geq 1$. Thus the function

$$
\mathcal{H}(x):=\|f\|_{L^{1}(S)}^{-2}|E f(x)|^{2} H(x)
$$

is a weight on $\mathbb{R}^{2}$ of dimension $\alpha^{\prime}=(\alpha / 2)+\epsilon$ and with

$$
A_{\alpha^{\prime}}(\mathcal{H}) \lesssim A_{\alpha}(H)\|f\|_{L^{1}(S)}^{-2}\|f\|_{L^{2}(S)}^{2} .
$$

Since $1<\alpha<2$, we have $1 / 2<\alpha^{\prime}<1$ provided $\epsilon$ is sufficiently small. So $Q\left(\alpha^{\prime}, 2\right) \leq 4 \alpha^{\prime}$, and so

$$
\int|E g(x)|^{q^{\prime}} \mathcal{H}(x) d x \lesssim A_{\alpha^{\prime}}(\mathcal{H})\|g\|_{L^{2}(S)}^{q^{\prime}}
$$

for all $g \in L^{2}(S)$ provided $q^{\prime}>4 \alpha^{\prime}=2 \alpha+4 \epsilon$. Replacing $\mathcal{H}$ by $\|f\|_{L^{1}(S)}^{-2}|E f|^{2} H$, plugging $f$ for $g$, and choosing $\epsilon$ to be sufficiently small, the last estimate becomes

$$
\int|E f(x)|^{q} H(x) d x \lesssim A_{\alpha}(H)\|f\|_{L^{2}(S)}^{q}
$$

for $q>2 \alpha+2$, which proves the inequality on the line next to the last in (27).

Now suppose $n \geq 3$ and $n / 2<\alpha \leq n$. Also, let $\epsilon>0,0<p \leq 2$, and $f \in L^{2}(S)$. Then Corollary 4-A and Hölder's inequality tell us that

$$
\begin{aligned}
\int_{B_{R}}|E f(x)|^{p} H(x) d x & \leq\left(C_{\epsilon} R^{\epsilon} A_{\alpha}(H) R^{\alpha / n}\|f\|_{L^{2}(S)}^{2}\right)^{p / 2}\left(\int_{B_{R}} H(x) d x\right)^{1-(p / 2)} \\
& \leq C_{\epsilon}^{p / 2} R^{p \epsilon / 2} A_{\alpha}(H)\|f\|_{L^{2}(S)}^{p} R^{(1-((n-1) p /(2 n)) \alpha}
\end{aligned}
$$

for all balls $B_{R} \subset \mathbb{R}^{n}$ of radius $R \geq 1$, which implies that the function $\mathcal{H}(x):=$ $\|f\|_{L^{1}(S)}^{-p}|E f(x)|^{p} H(x)$ is a weight on $\mathbb{R}^{n}$ of dimension

$$
\alpha^{\prime}=\left(1-\frac{n-1}{2 n} p\right) \alpha+\frac{p \epsilon}{2}
$$

and with

$$
A_{\alpha^{\prime}}(\mathcal{H}) \lesssim A_{\alpha}(H)\|f\|_{L^{1}(S)}^{-p}\|f\|_{L^{2}(S)}^{p} .
$$

Motivated by what we did in the plane, we want to choose a $p \in(0,2]$ that will place $\alpha^{\prime}$ between $(n-1) / 2$ and $n / 2$ and minimize the exponent $q_{0}$ given by

$$
q_{0}=\frac{4 \alpha^{\prime}}{n-1}+p=\frac{4 \alpha}{n-1}+\left(1-\frac{2 \alpha}{n}\right) p+\frac{2 p \epsilon}{n-1}
$$

Since $1-(2 \alpha / n)<0, q$ is smallest when $p$ is largest. Also, since $\epsilon$ can be chosen arbitrarily small,

$$
\frac{n-1}{2} \leq \alpha^{\prime} \leq \frac{n}{2} \Longleftarrow \frac{n-1}{2} \leq\left(1-\frac{n-1}{2 n} p\right) \alpha<\frac{n}{2} .
$$

Therefore,

$$
p=\frac{2 n}{n-1}-\frac{n}{\alpha}
$$

We note that $p \leq 2$ if $\alpha \leq n(n-1) / 2$, which is satisfied because $\alpha \leq n$ and $n \geq 3$. 
Since $(n-1) / 2 \leq \alpha^{\prime} \leq n / 2$, we now have $Q\left(\alpha^{\prime}, 2\right) \leq 4 \alpha^{\prime} /(n-1)$, and so

$$
\int|E g(x)|^{q^{\prime}} \mathcal{H}(x) d x \lesssim A_{\alpha^{\prime}}(\mathcal{H})\|g\|_{L^{2}(S)}^{q^{\prime}}
$$

for all $g \in L^{2}(S)$ provided $q^{\prime}>\left(4 \alpha^{\prime} /(n-1)\right)=q_{0}-p$. Replacing the weight $\mathcal{H}$ by $\|f\|_{L^{1}(S)}^{-p}|E f|^{p} H$, plugging $f$ for $g$, and choosing $\epsilon$ to be sufficiently small, the last estimate becomes

$$
\int|E f(x)|^{q} H(x) d x \lesssim A_{\alpha}(H)\|f\|_{L^{2}(S)}^{q}
$$

for $q>(2 n /(n-1))+2-(n / \alpha)$, proving the inequality on the last line of (27).

\section{Preliminaries for the proofs of Theorems 2.2 and 2.3}

Let $M\left(\mathbb{R}^{n}\right)$ be the space of all complex Borel measures on $\mathbb{R}^{n}$. Suppose $\mu \epsilon$ $M\left(\mathbb{R}^{n}\right)$ is positive and compactly supported, and $0<\alpha<n$. The $\alpha$-dimensional energy of $\mu$ is defined as

$$
I_{\alpha}(\mu)=\iint \frac{1}{|x-y|^{\alpha}} d \mu(x) d \mu(y)
$$

The integral $I_{\alpha}(\mu)$ has the following Fourier representation

$$
I_{\alpha}(\mu)=c_{\alpha} \int|\widehat{\mu}(\xi)|^{2} \frac{d \xi}{|\xi|^{n-\alpha}}=c_{\alpha} \int_{0}^{\infty}\|\widehat{\mu}(R \cdot)\|_{L^{2}\left(\mathbb{S}^{n-1}\right)}^{2} R^{\alpha-1} d R
$$

where $c_{\alpha}$ is a constant that only depends on $\alpha$ and $n$, and $\mathbb{S}^{n-1}$ is the unit sphere in $\mathbb{R}^{n}$.

For positive $\mu \in M\left(\mathbb{R}^{n}\right)$ and $0<\alpha<n$, we also define

$$
\mathcal{C}_{\alpha}(\mu)=\sup _{x \in \mathbb{R}^{n}, r>0} \frac{\mu(B(x, r))}{r^{\alpha}} .
$$

Let $1 \leq p \leq \infty$ and $p^{\prime}$ be the exponent conjugate to $p$. We want to establish a connection between $L^{p}(S) \rightarrow L^{q}\left(\chi_{B(0, R)} H d x\right)$ restriction estimates and the decay properties of $\|\widehat{\mu}(R \cdot)\|_{L^{p^{\prime}}(S)}$ as $R \rightarrow \infty$ for the positive measures $\mu \in M\left(\mathbb{R}^{n}\right)$ that are supported in the unit ball in $\mathbb{R}^{n}$ and satisfy $I_{\alpha}(\mu)<\infty$ or $\mathcal{C}_{\alpha}(\mu)<\infty$.

Proposition 8.1. Suppose $1 \leq p \leq \infty, q \geq 1,0<\alpha<n$, and we have the weighted local restriction estimate

$$
\int_{B(0, R)}|E f(x)|^{q} H(x) d x \leq C_{\epsilon} R^{\epsilon} A_{\alpha}(H)\|f\|_{L^{p}(S)}^{q} .
$$

Then

$$
\|\widehat{\mu}(R \cdot)\|_{L^{p^{\prime}(S)}} \leq C_{\epsilon} R^{\epsilon} \mathcal{C}_{\alpha}(\mu) R^{-\alpha / q} \quad(R \geq 1)
$$

for all positive measures $\mu \in M\left(\mathbb{R}^{n}\right)$ that are supported in $B(0,1)$. Moreover, if $q \geq 2$, then

$$
\|\widehat{\mu}(R \cdot)\|_{L^{p^{\prime}(S)}} \leq C_{\epsilon} R^{\epsilon} \sqrt{I_{\alpha}(\mu)} R^{-\alpha / q} \quad(R \geq 1)
$$

for all positive measures $\mu \in M\left(\mathbb{R}^{n}\right)$ that are supported in $B(0,1)$.

Proposition 8.1 is a standard result, which we state and prove here for clarity of exposition, as well as for highlighting the difference between the cases $1 \leq q<2$ and $q \geq 2$. The proof also reveals that the result of the proposition does not extend to the $0<q<1$ case, which is the main reason why Theorem 2.3 is much harder to prove than Theorem 2.2 . 
For the proof of Proposition 8.1 we need to borrow the following two lemmas from [17] and [22].

Lemma 8-A ([17, Lemma 5.1]). Suppose $\mu \in M\left(\mathbb{R}^{n}\right)$ is positive and supported in $B(0,1), 0<\alpha \leq n, R \geq 1$, and

$$
\mathcal{C}_{\alpha, R}(\mu)=\sup _{x \in \mathbb{R}^{n}} \sup _{r \geq R^{-1}} \frac{\mu(B(x, r))}{r^{\alpha}} .
$$

Then there is a weight $H$ (which depends on $R$ ) of dimension $\alpha$ such that:

(i) $A_{\alpha}(H) \leq|B(0,1)|$.

(ii) To every function $f \in L^{1}(S)$ there is a function $g \in L^{1}(S)$ such that $|g| \leq|f|$ and

$$
\int|E f(R x)|^{q} d \mu(x) \leq C_{q} \frac{\mathcal{C}_{\alpha, R}(\mu)}{R^{\alpha}} \int_{B(0,2 R)}|E g(y)|^{q} H(y) d y
$$

for $q \geq 1$, where $C_{q}$ is a constant that only depends on $n$ and $q$.

Lemma 8-B ([22, Lemma 1.5]). Let $\mu \in M\left(\mathbb{R}^{n}\right)$ be a positive measure with support in $B(0,1), 0<\alpha<n$, and $R \geq 1$. Then we can decompose $\mu$ as a sum of $O(1+\log R)$ measures $\mu_{j}$ so that for each $j$,

$$
\left\|\mu_{j}\right\| \mathcal{C}_{\alpha, R}\left(\mu_{j}\right) \lesssim I_{\alpha}(\mu)
$$

with an implicit constant that depends only on $\alpha$ and $n$.

Proof of Proposition 8.1. Let $f \in L^{1}(S)$, and $g$ be as in (ii) of Lemma 8-A. Then the weighted restriction estimate in the assumption of Proposition 8.1 tells us that

$$
\int|E f(R x)|^{q} d \mu(x) \leq C_{q} \frac{\mathcal{C}_{\alpha, R}(\mu)}{R^{\alpha}} C_{\epsilon}(2 R)^{\epsilon} A_{\alpha}(H)\|g\|_{L^{p}(S)}^{q},
$$

so that

$$
\int|E f(R x)|^{q} d \mu(x) \lesssim R^{\epsilon} \frac{\mathcal{C}_{\alpha, R}(\mu)}{R^{\alpha}}\|f\|_{L^{p}(S)}^{q},
$$

where we have used the facts that $A_{\alpha}(H) \leq|B(0,1)|$ and $|g| \leq|f|$ provided to us by Lemma $8-\mathrm{A}$

Since $q \geq 1$, we can use Hölder's inequality to get

$$
\left(\int|E f(R x)| d \mu(x)\right)^{q} \lesssim R^{\epsilon}\|\mu\|^{q-1} \frac{\mathcal{C}_{\alpha, R}(\mu)}{R^{\alpha}}\|f\|_{L^{p}(S)}^{q} .
$$

Since $\mu$ is supported in the unit ball, we have $\|\mu\|=\mu(B(0,1)) \leq \mathcal{C}_{\alpha, R}(\mu)$, so $\|\mu\|^{q-1} \mathcal{C}_{\alpha, R}(\mu) \leq \mathcal{C}_{\alpha, R}(\mu)^{q}$, and so

$$
\int|E f(R x)| d \mu(x) \lesssim R^{\epsilon / q} \frac{\mathcal{C}_{\alpha, R}(\mu)}{R^{\alpha / q}}\|f\|_{L^{p}(S)} .
$$

Since $E f=\widehat{f d \sigma}$, it follows that

$$
\left|\int \widehat{\mu}(R \xi) f(\xi) d \sigma(\xi)\right| \lesssim R^{\epsilon / q} \frac{\mathcal{C}_{\alpha, R}(\mu)}{R^{\alpha / q}}\|f\|_{L^{p}(S)}
$$

for all $f \in L^{p}(S)$. By duality, this implies that

$$
\|\widehat{\mu}(R \cdot)\|_{L^{p^{\prime}(S)}} \leq C_{\epsilon} R^{\epsilon / q} \mathcal{C}_{\alpha, R}(\mu) R^{-\alpha / q} \leq C_{\epsilon} R^{\epsilon} \mathcal{C}_{\alpha}(\mu) R^{-\alpha / q}
$$

for all $R \geq 1$. 
Now suppose $q \geq 2$ and write $\mu=\sum_{j} \mu_{j}$ as in Lemma 8-B. By Hölder's inequality, we have

$$
\begin{aligned}
\int|E f(R x)| d \mu_{j}(x) & \leq\left\|\mu_{j}\right\|^{1-(1 / q)}\left(\int|E f(R x)|^{q} d \mu_{j}(x)\right)^{1 / q} \\
& =\left\|\mu_{j}\right\|^{1-(2 / q)}\left(\left\|\mu_{j}\right\| \int|E f(R x)|^{q} d \mu_{j}(x)\right)^{1 / q} .
\end{aligned}
$$

Since $q \geq 2$, we have $\left\|\mu_{j}\right\|^{1-(2 / q)} \leq\|\mu\|^{1-(2 / q)}$. Also, by applying (29) to $\mu_{j}$ and then using the inequality $\left\|\mu_{j}\right\| \mathcal{C}_{\alpha, R}\left(\mu_{j}\right) \lesssim I_{\alpha}(\mu)$ from Lemma 8 -B we have

$$
\left\|\mu_{j}\right\| \int|E f(R x)|^{q} d \mu_{j}(x) \lesssim R^{\epsilon}\left\|\mu_{j}\right\| \frac{\mathcal{C}_{\alpha, R}\left(\mu_{j}\right)}{R^{\alpha}}\|f\|_{L^{p}(S)}^{q} \lesssim R^{\epsilon} \frac{I_{\alpha}(\mu)}{R^{\alpha}}\|f\|_{L^{p}(S)}^{q} .
$$

Therefore,

$$
\int|E f(R x)| d \mu_{j}(x) \lesssim\|\mu\|^{1-(2 / q)}\left(R^{\epsilon} \frac{I_{\alpha}(\mu)}{R^{\alpha}}\|f\|_{L^{p}(S)}^{q}\right)^{1 / q}
$$

Summing over $j$, this gives

$$
\int|E f(R x)| d \mu(x) \lesssim(1+\log R) R^{\epsilon / q}\|\mu\|^{1-(2 / q)} I_{\alpha}(\mu)^{1 / q} R^{-\alpha / q}\|f\|_{L^{p}(S)} .
$$

Since supp $\mu \subset B(0,1)$, we have $\|\mu\|^{2} \lesssim I_{\alpha}(\mu)$, and the above estimate becomes

$$
\int|E f(R x)| d \mu(x) \lesssim(1+\log R) R^{\epsilon / q} I_{\alpha}(\mu)^{1 / 2} R^{-\alpha / q}\|f\|_{L^{p}(S)} .
$$

Therefore,

$$
\left|\int \widehat{\mu}(R \xi)\right| f(\xi) d \sigma(\xi) \mid \lesssim R^{\epsilon} I_{\alpha}(\mu)^{1 / 2} R^{-\alpha / q}\|f\|_{L^{p}(S)}
$$

for all $f \in L^{p}(S)$, and the desired inequality, i.e.

$$
\|\widehat{\mu}(R \cdot)\|_{L^{p^{\prime}(S)}} \leq C_{\epsilon} R^{\epsilon} \sqrt{I_{\alpha}(\mu)} R^{-\alpha / q}
$$

for all $R \geq 1$, follows from duality.

We now need to complement Proposition 8.1 with some of the facts that we currently know about the decay properties of $\|\widehat{\mu}(R \cdot)\|_{L^{1}(S)}$ and $\|\widehat{\mu}(R \cdot)\|_{L^{p^{\prime}(S)}}$ when $S$ is the unit sphere. The first fact is the following basic result in geometric measure theory.

Proposition 8.2. Let $0<\alpha<n$. Then to every pair $(\beta, b)$ of numbers that satisfy $\beta>\alpha / 2$ and $b>0$ there is a number $R \geq 1$ and a positive measure $\mu \in M\left(\mathbb{R}^{n}\right)$ with $\operatorname{supp} \mu \subset B(0,1)$ such that $R^{\beta}\|\widehat{\mu}(R \cdot)\|_{L^{2}\left(\mathbb{S}^{n-1}\right)}>b \mathcal{C}_{\alpha}(\mu)$.

Proof. Suppose the proposition is not true. Then there is a pair $(\beta, b)$ with $\beta>\alpha / 2$ and $b>0$ such that $\|\widehat{\mu}(R \cdot)\|_{L^{2}\left(\mathbb{S}^{n-1}\right)} \leq b R^{-\beta} \mathcal{C}_{\alpha}(\mu)$ for all $R \geq 1$ and positive $\mu \in M\left(\mathbb{R}^{n}\right)$ that are supported in $B(0,1)$.

We let $\gamma<n$ be a number that lies strictly between $\alpha$ and $2 \beta$, and $K \subset B(0,1)$ be a set of Hausdorff dimension strictly between $\alpha$ and $\gamma$. Then $K$ carries a probability measure $\mu$ with $\mathcal{C}_{\alpha}(\mu)<\infty$. By the previous paragraph, we have $\|\widehat{\mu}(R \cdot)\|_{L^{2}\left(\mathbb{S}^{n-1}\right)} \lesssim R^{-\beta}$ for all $R \geq 1$, so (by (28) $) I_{\gamma}(\mu)<\infty$, and so $K$ carries a probability measure $\nu$ such that $\mathcal{C}_{\gamma}(\nu)<\infty$. This implies that $K$ has Hausdorff dimension $\geq \gamma$, which is a contradiction.

The second fact that complements Proposition 8.1 is due to Wolff [22]: 
Proposition 8-A ([22, Lemma 3.1]). Let $0<\alpha<n$. Then to every pair $(\beta, b)$ of numbers that satisfy $\beta>\alpha / 2$ and $b>0$ there is a number $R \geq 1$ and a positive measure $\mu \in M\left(\mathbb{R}^{n}\right)$ with $\operatorname{supp} \mu \subset B(0,1)$ such that $R^{\beta}\|\widehat{\mu}(R \cdot)\|_{L^{1}\left(\mathbb{S}^{n-1}\right)}>b \sqrt{I_{\alpha}(\mu)}$.

Proof. Let $\psi$ be a non-negative $C^{\infty}$ function on $\mathbb{R}^{n}$ that is supported in the unit ball and satisfies $|\widehat{\psi}| \geq 1$ on the unit sphere. For $0<\rho \leq 1$ and $x \in \mathbb{R}^{n}$, we let $\Psi(x)=\rho^{(\alpha / 2)-n} \psi\left(\rho^{-1} x\right)$, and we define the measure $\mu$ by $d \mu=\Psi d x$. Then $\widehat{\mu}(\xi)=\rho^{(\alpha / 2)} \widehat{\psi}(\rho \xi)$ and (by (28) $)$

$$
I_{\alpha}(\mu)=c_{\alpha} \rho^{\alpha} \int|\widehat{\psi}(\rho \xi)|^{2}|\xi|^{\alpha-n} d \xi=c_{\alpha} \int|\widehat{\psi}(u)|^{2}|u|^{\alpha-n} d u \sim 1 .
$$

Suppose the proposition is not true. Then there are numbers $\beta>\alpha / 2$ and $b>0$ such that $\|\widehat{\mu}(R \cdot)\|_{L^{1}\left(\mathbb{S}^{n-1}\right)} \leq b R^{-\beta} I_{\alpha}(\mu)$ for all $R \geq 1$, so that

$$
\int_{\mathbb{S}^{n-1}}|\widehat{\psi}(\rho R \theta)| d \sigma(\theta) \lesssim R^{-\beta} \rho^{-\alpha / 2}
$$

for all $0<\rho \leq 1$ and $R \geq 1$. Taking $R=\rho^{-1}$, we get

$$
\sigma\left(\mathbb{S}^{n-1}\right) \leq \int_{\mathbb{S}^{n-1}}|\widehat{\psi}(\theta)| d \sigma(\theta) \lesssim R^{-\beta} R^{\alpha / 2}
$$

for all $R \geq 1$, which implies that $\beta \leq \alpha / 2$, which is a contradiction.

The third fact that we need to complement Proposition 8.1 is the following result of Bennett and Vargas [2].

Theorem 8-A ([2, Corollary 2]). Let $1 \leq \alpha<2$. Then to every pair $(\beta, b)$ of numbers that satisfy $\beta>\alpha /(\alpha+2)$ and $b>0$ there is a positive measure $\mu \in M\left(\mathbb{R}^{2}\right)$ with $\operatorname{supp} \mu \subset B(0,1)$ such that $R^{\beta}\|\widehat{\mu}(R \cdot)\|_{L^{1}\left(\mathbb{S}^{1}\right)}>b \sqrt{I_{\alpha}(\mu)}$.

For the interesting proof of Theorem $8-\mathrm{A}$, we refer the reader to [2].

\section{Proof of Theorems 2.2}

We are given the estimate

$$
\int_{B_{R}}|E f(x)|^{q} H(x) d x \leq C_{\epsilon} R^{\epsilon} A_{\alpha}(H)\|f\|_{L^{2}(S)}^{q}
$$

for some $q>0$, and we need to show that

$$
q \geq \begin{cases}2 & \text { if } n \geq 2 \text { and } 0<\alpha<n, \\ (2 \alpha+2) /(n-1) & \text { if } n \geq 2 \text { and } 1<\alpha \leq n, \\ 4 \alpha & \text { if } n=2 \text { and } 1 / 2 \leq \alpha \leq 1 .\end{cases}
$$

In fact, the first line of (31) proves the first and third lower bounds on $q$ in Theorem 2.2. the second line of (31) proves the second lower bound on $q$ in Theorem 2.2, and the third line of (31) is identical to the fourth lower bound on $q$ in Theorem 2.2.

In proving (31), we proceed backwards starting with the inequality on its last line.

Suppose $n=2$ and $1 / 2 \leq \alpha \leq 1$. We let $b=1-\alpha$ and define

$$
X_{b}=\left\{(x, y) \in \mathbb{R}^{2}: x>0 \text { and } 0 \leq y \leq x^{-b}\right\} .
$$


Recall from Subsection 3.4 that the characteristic function of $X_{b}$ is a weight on $\mathbb{R}^{2}$ of dimension $1-b=\alpha$, and $A_{\alpha}\left(\chi_{X_{b}}\right) \lesssim 1$. So (30) implies that

$$
\int_{X_{b} \cap B_{R}}|E f(x)|^{q} d x \lesssim R^{\epsilon}\|f\|_{L^{2}(S)}^{q}
$$

for all $R \geq 1$. We now use the same Knapp-example argument that we used in the Introduction.

To every $R>1$ there is a function $f_{R}$ on $S$ such that $\left\|f_{R}\right\|_{L^{2}(S)} \lesssim R^{-1 / 4}$ and $\left|E f_{R}\right| \gtrsim R^{-1 / 2}$ on the rectangle $[0, R] \times[0, \sqrt{R}]$. The intersection of this rectangle with $X_{b} \cap B(0, R)$ contains the rectangle $[0, R] \times\left[0, R^{-b}\right]$, so $\left\|E f_{R}\right\|_{L^{q}\left(X_{b} \cap B(0, R)\right)}^{q}$ $\gtrsim R^{(-q / 2)+\alpha}$, and so $R^{(-q / 2)+\alpha} \leq R^{\epsilon} R^{-q / 4}$. Therefore, $q \geq 4 \alpha$.

Moving to the second line of (31), we now suppose that $n \geq 2$ and $1<\alpha \leq n$. We let $b=(\alpha-1) /(n-1)$ and define

$$
\Omega_{b}=\cup_{l=1}^{\infty} \mathbb{R} \times\left[l^{1 / b}, 1+l^{1 / b}\right]^{n-1}
$$

and we observe that $0<b \leq 1$ and the characteristic function of $\Omega_{b}$ is a weight on $\mathbb{R}^{n}$ of dimension $1+(n-1) b=\alpha$ and with $A_{\alpha}\left(\chi_{\Omega_{b}}\right) \lesssim 1$. So (30) (applied with $\left.H=\chi_{\Omega_{b}}\right)$ implies that

$$
\int_{\Omega_{b} \cap B_{R}}|E f(x)|^{q} d x \lesssim R^{\epsilon}\|f\|_{L^{2}(S)}^{q}
$$

for all $R \geq 1$.

To every $R>1$, there is a function $f_{R}$ on $S$ satisfying $\left\|f_{R}\right\|_{L^{2}(S)} \lesssim R^{-(n-1) / 4}$ and $\left|E f_{R}\right| \gtrsim R^{-(n-1) / 2}$ on $[0, R] \times\left[l^{1 / b}, 1+l^{1 / b}\right]^{n-1}$ whenever $l^{1 / b} \leq \sqrt{R}$. Since there are $\sim R^{(n-1) b / 2}$ such boxes, we see that $\left\|E f_{R}\right\|_{L^{q}\left(\Omega_{b} \cap B(0, R)\right)} \gtrsim R^{m}$ with

$$
m=-\frac{n-1}{2}+\left(1+\frac{(n-1) b}{2}\right) \frac{1}{q} \text {. }
$$

We have $R^{m q} \lesssim R^{\epsilon} R^{-(n-1) q / 4}$ for all $R>1$, so $m \leq-(n-1) / 4$, and it follows that $(n-1) q \geq 2 \alpha+2$.

Suppose $n \geq 2$ and $0<\alpha<n$. We will prove the first inequality in (31) by contradiction. Assume $q<2$. Then the estimate (30) holds with $q$ replaced by an exponent $q_{0}$ that satisfies $q<q_{0}<2$ and $q_{0} \geq 1$ (and $C_{\epsilon}$ replaced by $C_{\epsilon} \sigma(S)^{\left(q_{0}-q\right) / 2}$ ). When we combine the resulting estimate with Proposition 8.1, we get the decay estimate

$$
\|\widehat{\mu}(R \cdot)\|_{L^{2}(S)} \leq C_{\epsilon}^{\prime} R^{\epsilon} \mathcal{C}_{\alpha}(\mu) R^{-\alpha / q_{0}} \quad(R \geq 1)
$$

for all positive measures $\mu \in M\left(\mathbb{R}^{n}\right)$ that are supported in $B(0,1)$. Proposition 8.2 now implies that $\alpha / q_{0} \leq \alpha / 2$, which implies that $q_{0} \geq 2$, which is a contradiction.

\section{Proof of Theorem 2.3}

We need to show that

$$
Q_{\mathrm{loc}}(\alpha, \infty) \geq \begin{cases}(n-1) / n & \text { if } n \geq 2 \text { and } 0<\alpha<n-1, \\ 2 \alpha /(n-1) & \text { if } n \geq 2 \text { and } 0<\alpha \leq n, \\ 3 \alpha & \text { if } n=2 \text { and } 0<\alpha \leq 1 \\ \alpha+2 & \text { if } n=2 \text { and } 1 \leq \alpha \leq 2\end{cases}
$$

In fact, the first line of (32) proves the first and third lower bound on $Q_{\text {loc }}(\alpha, \infty)$ in Theorem 2.3, the second line of (32) proves the second lower bound on $Q_{\text {loc }}(\alpha, \infty)$ 
in Theorem 2.3, the third line of (32) proves the fourth lower bound on $Q_{\text {loc }}(\alpha, \infty)$ in Theorem 2.3. and the last line of (32) proves the fifth lower bound on $Q_{\text {loc }}(\alpha, \infty)$ in Theorem 2.3

In proving (32), we proceed backwards starting with the inequality on its last line.

Suppose that $n=2$ and $1 \leq \alpha \leq 2$, and that we have the estimate

$$
\int_{B_{R}}|E f(x)|^{r} H(x) d x \leq C_{\epsilon} R^{\epsilon} A_{\alpha}(H)\|f\|_{L^{\infty}(S)}^{r}
$$

for some $r>0$. We need to prove that $r \geq \alpha+2$. We will do this by showing that $r<\alpha+2$ leads to a contradiction.

Suppose (33) holds for some $r<\alpha+2$. We let $q$ be an exponent that satisfies $r<q<\alpha+2$ and $q \geq 2$. Then (33) holds with $r$ replaced by $q$ and $C_{\epsilon}$ replaced by $\sigma(S)^{q-r} C_{\epsilon}$. Since $q \geq 2$, it follows by Proposition 8.1 that

$$
\|\widehat{\mu}(R \cdot)\|_{L^{1}(S)} \leq C_{\epsilon}^{\prime} R^{\epsilon} \sqrt{I_{\alpha}(\mu)} R^{-\alpha / q} \quad(R \geq 1)
$$

for all positive measures $\mu \in M\left(\mathbb{R}^{n}\right)$ that are supported in $B(0,1)$. By Theorem 8-A it then follows that $\alpha / q \leq \alpha /(\alpha+2)$, which implies that $q \geq \alpha+2$, which is a contradiction.

We now move to the inequality before the last in (32). So we are still in the plane, but now $0<\alpha \leq 1$. We have just proved that $Q_{\text {loc }}(1, \infty) \geq 3$, so, by Corollary 2.1 . we have

$$
\frac{Q_{\mathrm{loc}}(\alpha, \infty)}{\alpha} \geq \frac{Q_{\mathrm{loc}}(1, \infty)}{1} \geq 3
$$

and so $Q_{\text {loc }}(\alpha, \infty) \geq 3 \alpha$.

Suppose that $n \geq 2$ and $0<\alpha \leq n$. The fact that $Q_{\text {loc }}(n, \infty) \geq 2 n /(n-1)$ follows from the fact that the $|\widehat{\sigma}(\xi)| \sim|\xi|^{-(n-1) / 2}$ for large $\xi$. Applying Corollary 2.1 as in the previous paragraph, we obtain the second inequality in (32).

The rest of the proof will be concerned with the first inequality in (32).

Suppose that $n \geq 2$ and $0<\alpha<n-1$, and that we have the estimate

$$
\int_{B_{R}}|E f(x)|^{r} H(x) d x \leq C_{\epsilon} R^{\epsilon} A_{\alpha}(H)\|f\|_{L^{\infty}(S)}^{r}
$$

for some $r>0$. We need to prove that $r \geq(n-1) / n$.

We apply the Cauchy-Schwarz inequality in (34) to get

$$
\begin{aligned}
\int_{B_{R}}|E f(x)|^{r / 2} H(x) d x & \leq\left(C_{\epsilon} R^{\epsilon} A_{\alpha}(H)\|f\|_{L^{\infty}(S)}^{r}\right)^{1 / 2}\left(\int_{B_{R}} H(x) d x\right)^{1 / 2} \\
& \leq C_{\epsilon}^{1 / 2} A_{\alpha}(H)\|f\|_{L^{\infty}(S)}^{r / 2} R^{\beta}
\end{aligned}
$$

for all balls $B_{R} \subset \mathbb{R}^{n}$ of radius $R \geq 1$, where $\beta=(\alpha+\epsilon) / 2$. This means $\mathcal{H}:=$ $\|f\|_{L^{1}(S)}^{-r / 2}|E f|^{r / 2} H$ is a weight of dimension $\beta$ with

$$
A_{\beta}(\mathcal{H}) \leq C_{\epsilon}^{1 / 2} A_{\alpha}(H)\|f\|_{L^{1}(S)}^{-r / 2}\|f\|_{L^{\infty}(S)}^{r / 2} .
$$

We have $0<\alpha / 2<(n-1) / 2$. So, from here on, we may assume that $\epsilon$ is small enough for us to have $0<\beta<(n-1) / 2$, which will allow us to apply part (ii) of Proposition 6.1 with any weight of dimension $\beta$. 
We let $B_{\rho}$ be a ball in $\mathbb{R}^{n}$ of radius $\rho \geq 1$, and we apply part (ii) of Proposition 6.1 with the weight $\chi_{B_{\rho}} \mathcal{H}$ to get

$$
\int_{B_{\rho}}|E f(x)|^{q} \mathcal{H}(x) d x \leq \bar{C}_{q}^{q} A_{\beta}(\mathcal{H})^{1 /(n-2 \beta)}\|\mathcal{H}\|_{L^{2}\left(B_{\rho}\right)}^{(n-1-2 \beta) /(n-2 \beta)}\|f\|_{L^{2}(S)}^{q}
$$

for $q>q_{0}$, where

$$
q_{0}=\frac{n+1-2 \beta}{n-2 \beta} .
$$

We already have the bound on $A_{\beta}(\mathcal{H})$ from the previous paragraph. Also, (34) tells us that to every $\delta^{\prime}>0$ there is a constant $C_{\delta^{\prime}}$ such that

$$
\|\mathcal{H}\|_{L^{2}\left(B_{\rho}\right)}^{2}=\|f\|_{L^{1}(S)}^{-r} \int_{B_{\rho}}|E f(x)|^{r} H(x)^{2} d x \leq\|f\|_{L^{1}(S)}^{-r} C_{\delta^{\prime}} \rho^{\delta^{\prime}} A_{\alpha}(H)\|f\|_{L^{\infty}(S)}^{r},
$$

where we have used the fact that $H^{2} \leq H$. So

$$
\begin{aligned}
\int_{B_{\rho}} & \|f\|_{L^{1}(S)}^{-r / 2}|E f(x)|^{q+(r / 2)} H(x) d x \\
\leq & \bar{C}_{q}^{q}\left(C_{\epsilon}^{1 / 2} A_{\alpha}(H)\|f\|_{L^{1}(S)}^{-r / 2}\|f\|_{L^{\infty}(S)}^{r / 2}\right)^{1 /(n-2 \beta)} \\
& \times\left(\|f\|_{L^{1}(S)}^{-r} C_{\delta^{\prime}} \rho^{\delta^{\prime}} A_{\alpha}(H)\|f\|_{L^{\infty}(S)}^{r}\right)^{(n-1-2 \beta) /(2(n-2 \beta))}\|f\|_{L^{2}(S)}^{q} \\
\leq & C_{q, \epsilon, \delta} \rho^{\delta} A_{\alpha}(H)^{(n+1-2 \beta) /(2(n-2 \beta))}\|f\|_{L^{1}(S)}^{-r / 2}\|f\|_{L^{\infty}(S)}^{r / 2}\|f\|_{L^{2}(S)}^{q}
\end{aligned}
$$

provided $q>(n+1-2 \beta) /(n-2 \beta)$, where $\delta=\delta^{\prime}(n-1-2 \beta) /(2(n-2 \beta))$, and so

$$
\int_{B_{\rho}}|E f(x)|^{q+(r / 2)} H(x) d x \leq C_{q, \epsilon, \delta} \rho^{\delta} A_{\alpha}(H)^{q_{0} / 2}\|f\|_{L^{2}(S)}^{q}\|f\|_{L^{\infty}(S)}^{r / 2} .
$$

We now let $\mu \in M\left(\mathbb{R}^{n}\right)$ be positive, supported in the unit ball $B(0,1)$, and satisfies $\mathcal{C}_{\alpha}(\mu)<\infty$. Since $q_{0}=(n+1-2 \beta) /(n-2 \beta)>1$, we have $q>1$, and so we can apply Lemma 8 -A (with $q+(r / 2)$ replacing $q$ ) to get a weight $H$ on $\mathbb{R}^{n}$ of dimension $\alpha$ that satisfies

- $A_{\alpha}(H) \leq|B(0,1)|$

- to every function $f \in L^{1}(S)$ there is a function $g \in L^{1}(S)$ such that $|g| \leq|f|$ and

$$
\int|E f(\rho x)|^{q+(r / 2)} d \mu(x) \leq C^{\prime \prime} \frac{\mathcal{C}_{\alpha}(\mu)}{\rho^{\alpha}} \int_{B(0,2 \rho)}|E g(y)|^{q+(r / 2)} H(y) d y,
$$

where $C^{\prime \prime}$ depends on $q, r$, and $n$.

Then (35) implies that

$$
\int|E f(\rho x)|^{q+(r / 2)} d \mu(x) \leq C^{\prime \prime \prime} \frac{\mathcal{C}_{\alpha}(\mu)}{\rho^{\alpha}} \rho^{\delta}\|f\|_{L^{2}(S)}^{q}\|f\|_{L^{\infty}(S)}^{r / 2} .
$$

Letting $\gamma=\alpha-\delta$ and $p=q+(r / 2)$, and using Hölder's inequality, this becomes

$$
\int|E f(\rho x)| d \mu(x) \leq C \frac{1}{\rho^{\gamma / p}}\|f\|_{L^{2}(S)}^{q / p}\|f\|_{L^{\infty}(S)}^{1-q / p} .
$$

Therefore,

$$
\left|\int \widehat{\mu}(\rho \xi) f(\xi) d \sigma(\xi)\right| \leq C \frac{1}{\rho^{\gamma / p}}\|f\|_{L^{2}(S)}^{q / p}\|f\|_{L^{\infty}(S)}^{1-(q / p)}
$$


We will use (36) to estimate the $\sigma$-measure of the set

$$
\{\xi \in S:|\widehat{\mu}(\rho \xi)|>\lambda\}
$$

for $0<\lambda \leq\|\mu\|$. For such $\lambda$ and for $l \in \mathbb{N}$, we set

$$
X_{l}=X_{l}(\lambda)=\left\{\xi \in S: 2^{l-1} \lambda<|\widehat{\mu}(\rho \xi)| \leq 2^{l} \lambda\right\} .
$$

Clearly,

$$
\{\xi \in S:|\widehat{\mu}(\rho \xi)|>\lambda\} \subset \cup_{l=1}^{\infty} X_{l} .
$$

Inserting $\overline{\widehat{\mu}(\rho \xi)} \chi_{X_{l}}(\xi)$ for $f(\xi)$ in (36), we obtain

$$
\left(\int_{X_{l}}|\widehat{\mu}(\rho \xi)|^{2} d \sigma(\xi)\right)^{1-(q /(2 p))} \leq C \rho^{-\gamma / p}\left(2^{l} \lambda\right)^{1-(q / p)},
$$

which implies that

$$
\left(\sigma\left(X_{l}\right)\left(2^{l-1} \lambda\right)^{2}\right)^{1-(q /(2 p))} \leq C \rho^{-\gamma / p}\left(2^{l} \lambda\right)^{1-(q / p)},
$$

which in turn implies that

$$
\sigma\left(X_{l}\right) \lesssim \rho^{-2 \gamma /(2 p-q)}\left(2^{l} \lambda\right)^{-2 p /(2 p-q)}
$$

for all $l \in \mathbb{N}$. Since $2 p-q=q+r$, we have $\sum_{l=1}^{\infty} 2^{-2 l p /(2 p-q)} \sim 1$, and hence

$$
\sigma(\{\xi \in S:|\widehat{\mu}(\rho \xi)|>\lambda\}) \leq \sum_{l=1}^{\infty} \sigma\left(X_{l}\right) \lesssim \rho^{-2 \gamma /(2 p-q)} \lambda^{-2 p /(2 p-q)} .
$$

Of course, we also have the trivial bound

$$
\sigma(\{\xi \in S:|\widehat{\mu}(R \xi)|>\lambda\}) \leq \sigma(S) \lesssim 1 .
$$

We now let $p_{0}=2 p /(2 p-q)$ and use the two bounds we now have on the $\sigma$ measure of the set $\{\xi \in S:|\widehat{\mu}(\rho \xi)|>\lambda\}$ to see that

$$
\begin{aligned}
\int_{0}^{\|\mu\|} & \sigma(\{\xi \in S:|\widehat{\mu}(\rho \xi)|>\lambda\}) \lambda^{p_{0}-1} d \lambda \\
& \lesssim \int_{0}^{\rho^{-\gamma / p}} \lambda^{p_{0}-1} d \lambda+\rho^{-\gamma p_{0} / p} \int_{\rho^{-\gamma / p}}^{\|\mu\|} \frac{d \lambda}{\lambda} \lesssim \rho^{-\gamma p_{0} / p} \log \rho
\end{aligned}
$$

provided $\rho \geq\left(\|\mu\|^{-1}+\|\mu\|\right)^{p / \gamma}$. Thus

$$
\int|\widehat{\mu}(\rho \xi)|^{p_{0}} d \sigma(\xi) \lesssim(\log \rho) \rho^{-\gamma p_{0} / p} .
$$

Since $p_{0} \leq 2$, it follows that

$$
\int|\widehat{\mu}(\rho \xi)|^{2} d \sigma(\xi) \leq\|\mu\|^{2-p_{0}} \int|\widehat{\mu}(\rho \xi)|^{p_{0}} d \sigma(\xi) \lesssim(\log \rho) \rho^{-\gamma p_{0} / p} .
$$

The inequality we just derived is true for all positive measures $\mu \in M\left(\mathbb{R}^{n}\right)$ that are supported in the unit ball and satisfy $\mathcal{C}_{\alpha}(\mu)<\infty$. So, by Proposition 8.2 . $\gamma p_{0} / p \leq \alpha$. Recalling that $\gamma=\alpha-\delta$, and letting $\delta \rightarrow 0$, we see that $p_{0} \leq p$.

Replacing $p_{0}$ by its value in terms of $p$ and $q$, we see that $2 p-q \geq 2$. Replacing $p$ by its value in terms of $q$ and $r$, this becomes $r \geq 2-q$. Since this is true for every $q>(n+1-2 \beta) /(n-2 \beta)$, it follows that

$$
r \geq 2-\frac{n+1-2 \beta}{n-2 \beta} .
$$


Recalling that $\beta=(\alpha+\epsilon) / 2$, and letting $\epsilon \rightarrow 0$, we arrive at

$$
r \geq 2-\frac{n+1-\alpha}{n-\alpha} .
$$

If $\alpha^{\prime} \leq \alpha$, then any weight $H$ on $\mathbb{R}^{n}$ of dimension $\alpha^{\prime}$ is also a weight of dimension $\alpha$. Moreover, $A_{\alpha}(H) \leq A_{\alpha^{\prime}}(H)$. So the given estimate (34) holds for all weights on $\mathbb{R}^{n}$ of dimension $\alpha^{\prime} \leq \alpha$, and so we can send $\alpha \rightarrow 0$ in (37) to get

$$
r \geq 2-\frac{n+1}{n}=\frac{n-1}{n},
$$

as promised.

Remark 10.1. During the proof of Theorem 2.3, we used the fact that $q+(r / 2)>1$ to obtain the decay estimate

$$
\|\widehat{\mu}(\rho \cdot)\|_{L^{2}(S)} \lesssim(\log \rho)^{1 / 2} \rho^{-\gamma p_{0} /(2 p)}
$$

for all positive measures $\mu \in M\left(\mathbb{R}^{n}\right)$ that are supported in the unit ball and satisfy $\mathcal{C}_{\alpha}(\mu)<\infty$. If, for some reason, we knew that $q+(r / 2) \geq 2$, then (35) (via Proposition 8.1) would give us the decay estimate

$$
\|\widehat{\mu}(\rho \cdot)\|_{L^{1}(S)} \lesssim \rho^{\epsilon} \rho^{-\alpha /(q+(r / 2))}
$$

for all positive measures $\mu \in M\left(\mathbb{R}^{n}\right)$ that are supported in the unit ball and satisfy $I_{\alpha}(\mu)<\infty$, which would have allowed us to use Proposition 8 -A to conclude that

$$
\frac{\alpha}{q+(r / 2)} \leq \frac{\alpha}{2}
$$

i.e. $r / 2 \geq 2-q$. Proceeding as we did in the last part of the proof of Theorem 2.3, we would have arrived at $r \geq 2(n-1) / n$.

\section{REFERENCES}

[1] J. Bourgain, Besicovitch type maximal operators and applications to Fourier analysis, Geom. Funct. Anal. 1 (1991), 147-187.

[2] J. Bennett And A. VARgas, Randomised circular means of Fourier transforms of measures, Proc. Amer. Math. Soc. 131 (2003), 117-127.

[3] C. Demeter, On the restriction theorem for paraboloid in $\mathbb{R}^{4}$, Colloq. Math. 156 (2019), 301-311.

[4] X. Du, L. Guth, Y. Ou, H. Wang, B. Wilson, and R. Zhang, Weighted restriction estimates and application to Falconer distance set problem, Amer. J. Math., to appear, arXiv:1802.10186.

[5] X. Du AND R. Zhang, Sharp $L^{2}$ estimate of Schrödinger maximal function in higher dimensions, Ann. of Math. 189 (2019), 837-861.

[6] M. B. ErdoĞAn, A note on the Fourier transform of fractal measures, Math. Res. Lett. 11 (2004), 299-313.

[7] M. B. ERdoĞAn, A bilinear Fourier extension theorem and applications to the distance set problem, Int. Math. Res. Not. 23 (2005), 1411-1425.

[8] J. KIM, Some remarks on Fourier restriction estimates, Preprint, arXiv:1702.01231

[9] L. Guth, A restriction estimate using polynomial partitioning, J. Amer. Math. Soc. 29 (2016), 371-413.

[10] L. Guth, Restriction estimates using polynomial partitioning II, Acta Math. 221 (2018), 81-142.

[11] L. Guth, J. Hickman, AND M. Iliopoulou, Sharp estimates for oscillatory integral operators via polynomial partitioning, Acta Math. 223 (2019), 251-376.

[12] T. L. J. HARRIS, Improved decay of conical averages of the Fourier transform, Proc. Amer. Math. Soc. 147 (2019), 4781-4796.

[13] J. Hickman, AND K. Rogers, Improved Fourier restriction estimates in higher dimensions, Camb. J. Math. 7 (2019), 219-282. 
[14] R. Lucì And K. Rogers, Average decay of the Fourier transform of measures with applications, J. Eur. Math. Soc. (JEMS) 21 (2019), 465-506.

[15] P. MatTiLA, Spherical averages of Fourier transforms of measures with finite energy: dimensions of intersections and distance sets, Mathematika 34 (1987), 207-228.

[16] T. Mitsis, A Stein-Tomas restriction theorem for general measures, Publ. Math. Debrecen 60 (2002), 89-99.

[17] B. SHAYYA, Weighted restriction estimates using polynomial partitioning, Proc. Lond. Math. Soc. (3) 115 (2017), 545-598.

[18] P. SנöLIn, Estimates of spherical averages of Fourier transforms and dimensions of sets, Mathematika 40 (1993), 322-330.

[19] T. TAO, The Bochner-Riesz conjecture implies the restriction conjecture, Duke Math. J. 96 (1999), 363-375.

[20] H. WANG, A restriction estimate in $\mathbb{R}^{3}$ using brooms, Preprint, arXiv:1802.04312.

[21] R. Wongkew, Volumes of tubular neighbourhoods of real algebraic varieties, Pacific J. Math. 159 (1993), 177-184.

[22] T. WolfF, Decay of circular means of Fourier transforms of measures, Int. Math. Res. Not. 10 (1999), 547-567.

[23] J. ZAHL, A discretized Severi-type theorem with applications to harmonic analysis, Geom. Funct. Anal. 28 (2018), 1131-1181.

Department of Mathematics, American University of Beirut, Beirut, Lebanon

Email address: bshayya@aub.edu.lb 